\title{
Nonlinear 3D finite element analysis of a shear-wave vibrator-ground interaction system
}

\author{
Xun Peng ${ }^{\mathrm{a}}$, Zhiqiang Huang ${ }^{\mathrm{a},}$, , Susana López-Querol ${ }^{\mathrm{b}}$, Lei Hao $^{\mathrm{c}}$ \\ ${ }^{a}$ Electromechanical Engineering College, Southwest Petroleum University, Chengdu, 610500, PR China \\ ${ }^{b}$ Department of Civil, Environmental and Geomatic Engineering, University College London, London, WC1E 6BT, \\ UK \\ ${ }^{\mathrm{c} B u r e a u}$ of Geophysical Prospecting, China National Petroleum Corporation, Zhuozhou, 072750, PR China \\ ${ }^{*}$ Corresponding author \\ Declarations of interest: none.
}

Abstract: Shear-wave vibrator is a type of equipment usually employed for natural gas exploration, consisting of a vibrating machine which applies dynamic loads to the surrounding soils in order to ease the extraction of the gas. Detailed numerical simulations of these devices are scarce in the literature. Thus, in this paper, a nonlinear 3D finite element numerical simulation has been conducted to investigate the main features of the dynamic response of the vibrator. Nonlinear aspects such as baseplates-soil interaction, soil plasticity, and non-reflect boundary conditions, usually neglected in previous simulations, are considered in this model. The stress distribution, plastic deformation, shear wave propagation, ground force and energy characteristics of the vibrator-ground system are analyzed in detail. Based on the achieved results, an evaluation criterion is developed to quantify the performance of the shear-wave vibrator. Moreover, a parametric analysis has been conducted and the effects of some system parameters, such as excitation frequency, different soil properties and embedded depth, are analyzed and discussed in detail. The results can provide a reference for operating or optimizing the design of shear-wave vibrators.

Keywords: s-wave vibrator; nonlinear interaction; finite element analysis; seismic exploration

\section{Introduction}

A seismic vibrator is a device that is typically used to send out sweep signals. Seismic vibrators apply dynamic energy to the ground. Under working conditions, these vibrations are the principal source of energy in land exploration [1,2]. Depending on the types of the exciting seismic load, the vibrator can be categorized into compressional-wave ( $p$-wave) or shear-wave (s-wave) vibrator $[3,4]$. In addition, due to serious environmental constraints, nowadays the demand for natural gas has increased because it is an environmental-friendly type of energy. Although p-wave vibrators can meet the requirements under normal usage for regular 
exploration conditions, a practical implementation of the p-wave exploration technique for soft soil may often be problematic. Since shear-waves have shorter wavelength (resulting in higher vertical resolution and manage to image through gas clouds), they are more effective way for natural gas prospection, so the application of s-wave seismic vibrators in geotechnical investigation seems to be more convenient.

The development of the s-wave seismic vibrator faces some difficulties. S-wave sources typically are limited to obtain sufficient detail in the resulting subsurface images, due to their usual frequency content. Meanwhile, the s-wave seismic vibrator can be much heavier than pwave devices to obtain the same source strength. In order to improve the accuracy and the effectiveness of a s-wave vibrator, it is important to study its response and the influence factors on its performance. Previous studies show that the dynamic properties of the vibrator-ground interaction system have great influence on the performance of the vibrator and the output signal [5-6]. Thus, it is necessary to find out an appropriate research method and analyze the dynamic response of the s-wave vibrator-ground system.

Various research efforts have been made in the past to study the dynamic response of the p-wave vibrator-ground system and how the system parameters affect its response. The main research methods are field tests, theoretical modelling, and finite-element simulations. Regarding the field research, many tests have been devoted to the distortion of the outgoing wave compared with the excitation signal and have discussed the impacts of different operation conditions [7-11]. In addition, to find out an efficient control method, some researchers have considered the theoretical model of the vibrator-ground system, mostly relying on linear behavior considerations [12-14]. Due to this limitation, many practical issues arising from these field tests remain theoretically unexplained, and they have typically been attributed to the nonlinearity in the complex vibrator-ground system. In order to study the influence of the nonlinear contacts on the performance of the system, Lebedev [15] and Beresnev and Lebedev et al. [16] propose a model with nonlinear contact springs. Noorlandt and Drijkoningen [17] and Huang et al. [18] established nonlinear theoretical models that consider the effects of the surface topography at the interfaces between the vibrator and ground. In addition, to account for the dynamics of the coupling ground, some researchers introduced dynamic stiffness and dynamic damping into the theoretical model to simulate the performance of the vibrator at the elastic half-space surface [19]. With the appearance of the nonlinear factors introduced into the theoretical model, it is difficult to find out an analytical solution for the model, or even approximate one. Meanwhile, most of the existing theoretical models lack of any consideration on the flexural behavior of the vibrator baseplate which can also affect the overall performance of the system [20]. Thus, the finite element analysis comes into light as an efficient tool to 
simulate the dynamics of the vibrator-ground system. As an example, Wei et al. and Huang et al. proposed a finite element model for a p-wave vibrator located on an elastic ground column [21-23]. By using this model, they studied the response of the vibrator with different excitation frequencies and soil properties. It is worth mentioning that in previous finite element analyses the influence of plasticity was fully neglected.

Although some field tests of s-wave vibrators can be found in the literature [24-25], to our best knowledge, no comprehensive theoretical models or finite element analyses have been reported so far to investigate the dynamics of the s-wave vibrator-ground interaction system. Due to the multiple nonlinear factors in these systems, such as soil plasticity and baseplatessoil interaction, it is difficult to develop a theoretical model taking into account all these factors. Moreover, the finite element method is widely used to model soil-structure interactions [26-30]. The soil-structure system, in which the interaction and soil plasticity have great influence, has similarities with the vibrator-ground system. Thus, finite element analysis seems to be an appropriate method to simulate the vibrator-ground system.

The main goal of this study is to propose an accurate nonlinear, three-dimensional finite element model that to simulate the interaction between the s-wave vibrator and the ground. The dynamic response of a s-wave vibrator under harmonic excitation is analyzed by using the presented model. Meanwhile, a criterion is established to evaluate the performance of the vibrator for different system parameters. Thus, the effects of the excitation frequency, soil properties and embedded depth on the s-wave vibrator performance are discussed.

\section{Description and methodology}

\subsection{Description of the s-wave vibrator}

The s-wave seismic vibrator usually generates seismic waves through the horizontal oscillation of the vibrator, as shown in Fig. 1. As the key component of the s-wave seismic source, the vibrator mainly consists of two parts: the top structure (including the box body, the reaction mass, and the piston) and the baseplates. Figure 1b) shows a sketch of the s-wave vibrator analyzed in the present research, where length, width and height are $2.37 \times 1.58 \times 1.12$ $\mathrm{m}$, respectively. The analyzed s-wave vibrator is mounted on an BV-300 vibroseis, and the dimension details are offered by the Bureau of Geophysical Prospecting of China. Normally, the contact between the vibrator baseplates and ground is simply generated by the weight of the vehicle (hold-down load) in the static loading phase. The drive system is then used to pump the hydraulic oil into the chamber between the reaction mass and piston and to apply a time-varying pressure to the piston. The excitation force is transferred to the baseplates through the piston 
and the box body and finally into the earth through the baseplates-soil interaction. The contact properties and deformation at the interface have great influence on the accuracy of the outgoing waves and the power of the vibrator which can determine the effectiveness of the s-wave vibrator. Thus, obtaining deformations and stresses in the contacts is the objective of this research, in order to assess the optimal effectiveness of this devices. Meanwhile, the shear stress wave generated by the vibrator represents the accuracy of the output signal which also needs to be studied.

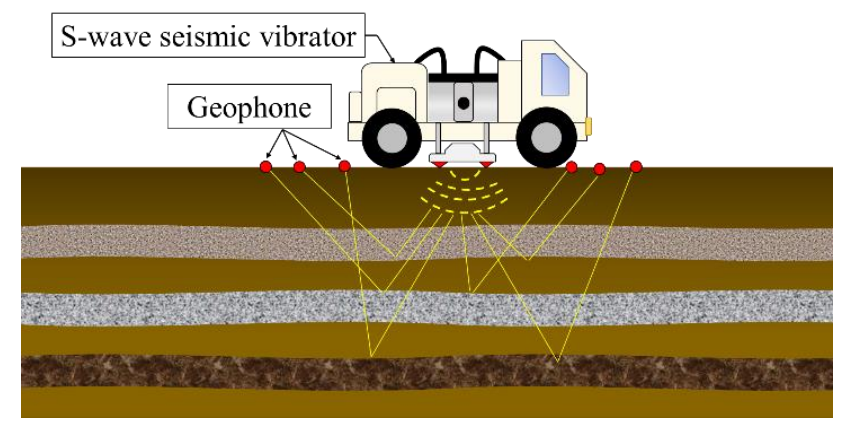

(a)

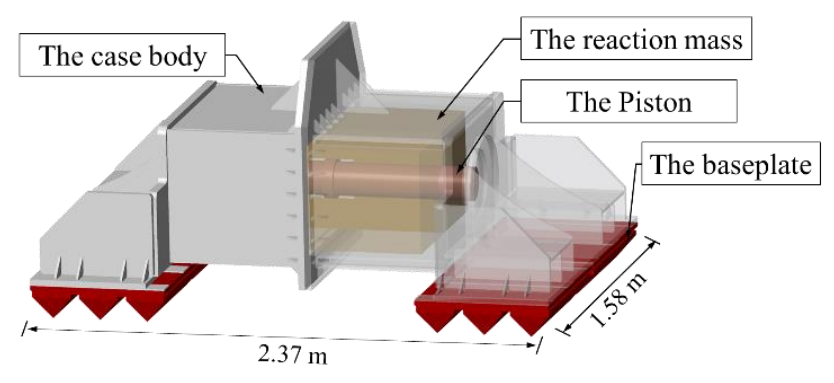

(b)

Figure 1. Schematic diagram of the s-wave seismic vibrator: (a) sketch of the s-wave vibrator; (b) the geometric model of the vibrator.

\subsection{Soil constitutive model}

In previous simulation models of p-wave vibrator-ground systems, the soil material was generally considered as completely elastic in past researches, as mentioned. The actual interaction between the s-wave vibrator and ground is more complicated than for p-wave vibrators, because the baseplates of the s-wave vibrators are introduced into the soil while the contact between the p-wave vibrator and ground can be simplified as a flat surface contact. The soil behavior can show nonlinear elastic-plastic properties and even a geometric nonlinearity caused by large strains. Thus, in this research, and as improvement respect previous models, the nonlinear soil behavior is considered to be governed by an elastic-perfectly plastic constitutive model according to the Drucker-Prager yield condition, which has been adopted in many finite element analyses of soil dynamics [31-33]. The yield function can be written as 


$$
F=\alpha I_{1}+\sqrt{J_{2}}-k=0
$$

where $F$ is the yield surface, $\alpha$ are $k$ are soil dependent positive constant values, $I_{1}$ is the first invariant of stress tensor, $J_{2}$ is the second invariant of deviatoric stress tensor:

$$
\begin{gathered}
I_{1}=\sigma_{1}+\sigma_{2}+\sigma_{3} \\
J_{2}=\frac{1}{6}\left[\left(\sigma_{1}-\sigma_{2}\right)^{2}+\left(\sigma_{2}-\sigma_{3}\right)^{2}+\left(\sigma_{3}-\sigma_{1}\right)^{2}\right]
\end{gathered}
$$

where $\sigma_{1}, \sigma_{2}$ and $\sigma_{3}$ are the principle stress of effective stress. When matching DruckerPrager criterion to Mohr-Coulomb criterion, the parameters $\alpha$ and $k$ are

$$
\alpha=\frac{2 \sin \varphi}{\sqrt{3}(3 \pm \sin \varphi)}, k=\frac{6 c \cos \varphi}{\sqrt{3}(3 \pm \sin \varphi)}
$$

where $c$ is the material cohesion, $\varphi$ is the angle of internal friction. Here, the positive and negative signs indicate the tensile and compressive conditions, respectively.

\subsection{Baseplates-soil interface}

Slippage and separation may occur in the interfaces between the baseplates and soil during the vibration process. Thus, the interface between baseplates and soil has a nonlinear behavior. In this research, it is defined by using master-slave kinematic contact algorithm. For the normal contact behavior, the surface transmits no contact pressure unless the nodes of the slave surface contact the master surface and no penetration is allowed at each contact location. Meanwhile, Coulomb friction model is employed to define the tangential motion, and the friction coefficient represents the friction characteristics between the contact interfaces [34]. The governing equation is:

$$
\tau_{\text {crit }}=\mu \times p
$$

where $\tau_{c r i t}$ is the critical shear stress, $\mu$ is the friction coefficient, and $p$ is the normal stress in the contact. When the shear stress in the contact is less than the critical shear stress, the state of contact surfaces is bonding, i.e. without relative movement.

\subsection{Far-field boundary condition}

As previously justified, the analyzed problem is based on the transmission of seismic waves generated by the horizontal vibration of the vibrator through the soil mass. If the usual fixed boundary conditions are applied at the space domain limits, the seismic waves are reflected into the calculation domain and introduce artificial vibration, while this reflection does not happen in the real field. In order to allow the seismic waves to leave the calculation domain and simulate infinite space, to replicate field conditions, it is necessary to use absorbing boundaries. The classic standard viscous boundary condition, presented by Lysmer and 
Kuhlemeyer [35], is able to perfectly absorb waves that propagate normally to the boundary by means of the application of a normal condition to a free artificial boundary, so that any reflected stresses are zero. This boundary condition is physically represented by a series of normal and tangential dashpots on the boundary which, for plane strain conditions, are described by

$$
\begin{gathered}
\sigma(t)=\rho V_{p} \frac{\partial u(t)}{\partial t} \\
\tau(t)=\rho V_{s} \frac{\partial v(t)}{\partial t}
\end{gathered}
$$

where $\sigma(t), \tau(t)$ respectively denote normal and shear stresses at the boundary, $u(t)$ and $v(t)$ are the normal and tangential displacements, $V_{p}$ is the compression wave velocity, $V_{s}$ represent the shear wave velocity of the medium, $t$ is the time and $\rho$ is the soil mass density.

\section{Numerical modelling process}

The dynamic response of the vibrator-ground system is examined by using finite element tools. 3D model of the vibrator-ground system is established considering the baseplates-soil interaction, soil plasticity and absorbing boundaries. By doing so, the results obtained with these models are expected to be closer to the engineering practice than simpler approximations. The details of the finite element model and results are discussed in the following sections. The research to aid the design of a vibrator-ground system is represented in Fig. 2.

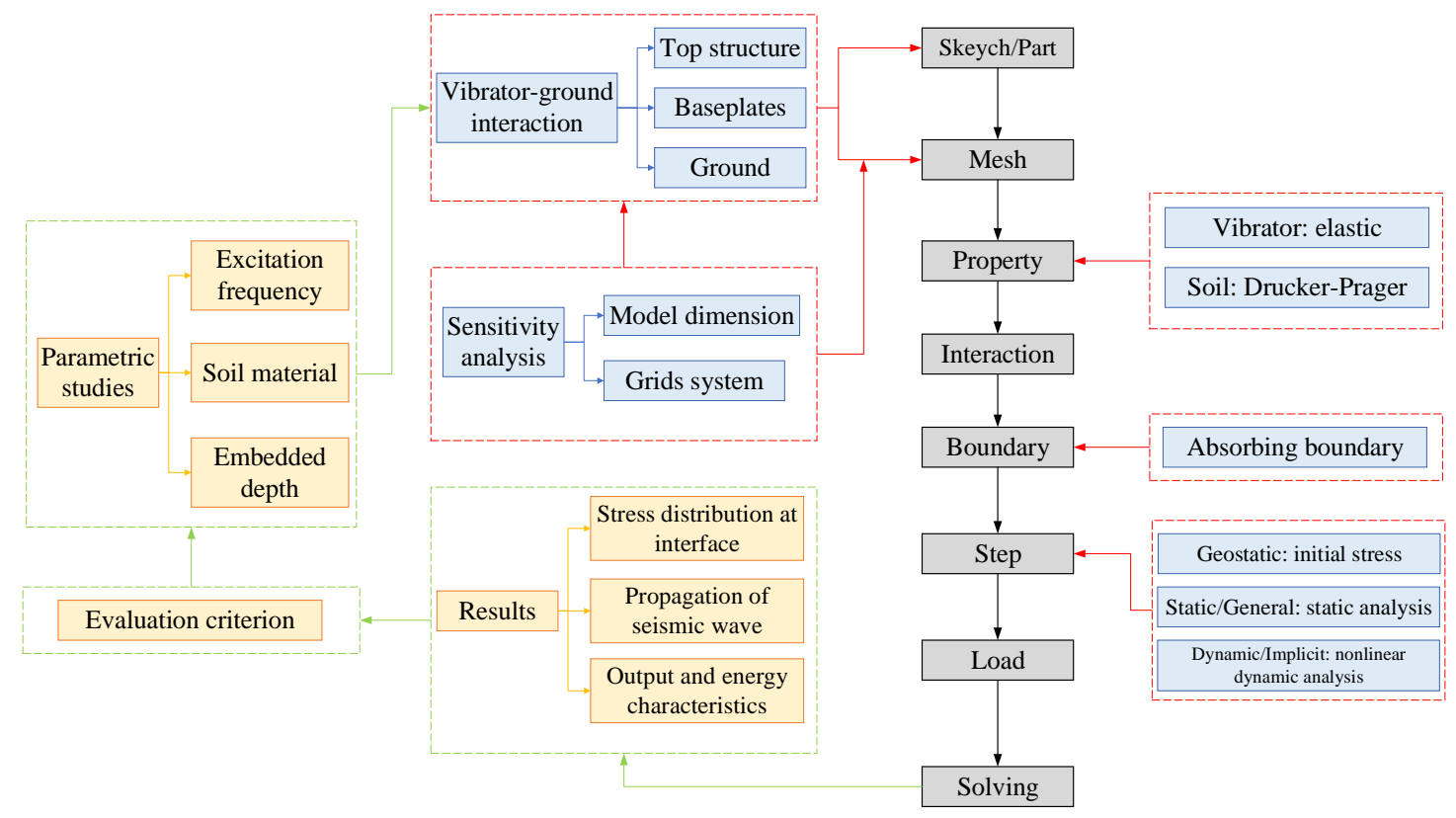

Figure 2. The research process of the vibrator-ground model

\subsection{Mesh details}

Since the model is primarily concerned with the interaction between the baseplate and soil, 
the dynamic pressure is applied on the piston directly (see Fig.1). Figure 3 represents the developed finite element model of the vibrator-ground system, which contains three distinct parts: (1) the top structure (including the box body and the piston), (2) the baseplates, (3) the ground column. The geometric model of the top structure is complex, and a large number of elements need to be generated to fully represent the geometry of the vibrator, which substantially increases the computational effort and calculation time. Thus, the top structure is represented as a discrete rigid body and a shell is extracted from it. The point mass and inertia properties are assigned by locating the rigid body reference node at the center of mass and by specifying the rigid body mass and rotary inertia at the reference node. The element type of the top structure is a 4-node bilinear rigid quadrilateral element, which dramatically reduces the size of the mesh. The baseplate and ground column are discretized by 8 -node linear brick elements with reduced integration and hourglass control [33]. Since the geometry and load of the model is symmetric about the $y-z$ plane, only one-half of the total region is considered.

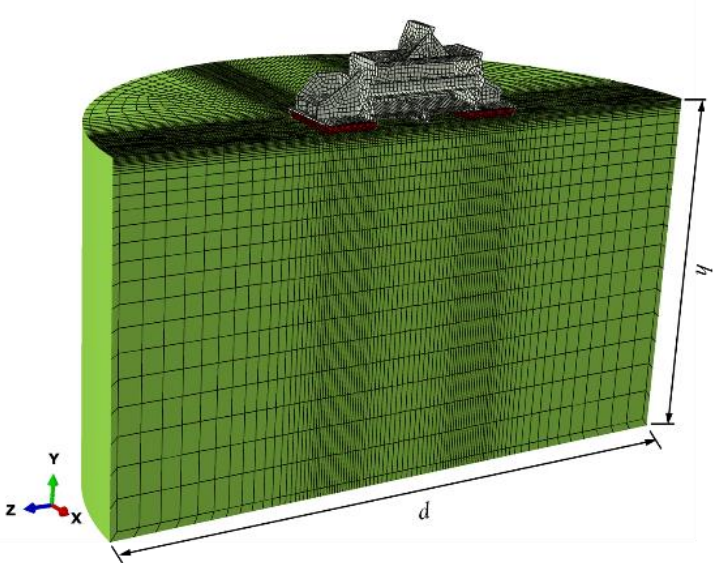

(a)

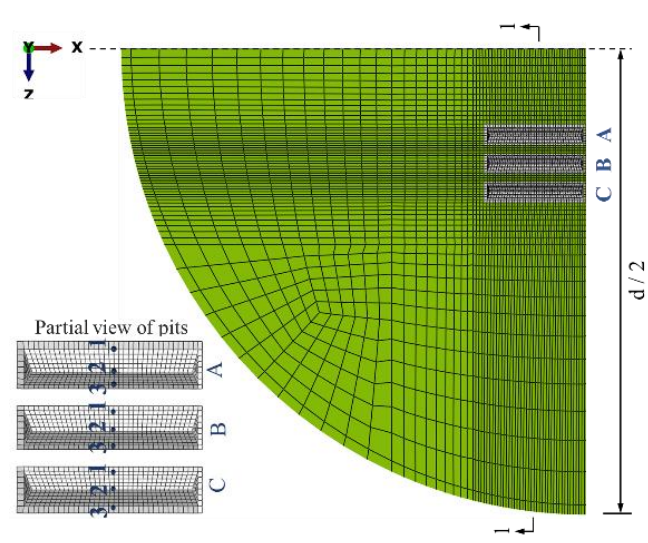

(b)

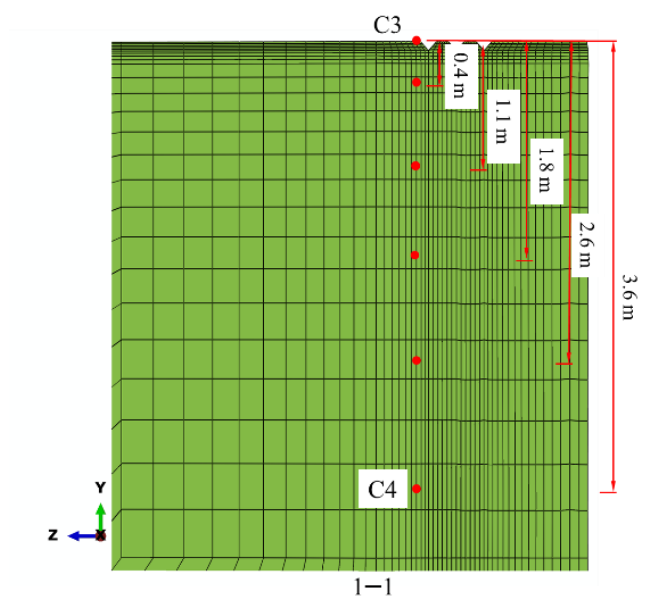

(c)

Figure 3. The mesh model of the vibrator-ground system: (a) 3D view of the mesh model; (b) top view of the left half of the ground model; (c) section view of the ground model. 
Primary tests show that a uniform mesh in the whole of the ground model generates a huge number of elements and accordingly increases the calculation time considerably. Therefore, the element size at the interfaces should have to be fine enough to maintain the convergence at the contacts, and the remaining region of the ground can be meshed with bigger size. The largest size of the elements should be limited to $1 / 5-1 / 4$ of the smallest wavelength for 8 -noded isoperimetric elements [36]. Analyses are performed with several trial meshes of the ground model with increasing refinement until the displacement at the interfaces does not significantly change with more refinement, as shown in Table. 1. The difference between Grid 1 and Grid 2 is much bigger than that between Grid 3 and Grid 4. Thus, to save computational resources and balance computational economy and prediction accuracy, Grid 3 is chosen in this analysis. Therefore, the finite elements mesh consists of approximately 17938 4-node elements for the top structure and 3361128 -node elements for the other parts of the system.

Table 1. Mesh independence test

\begin{tabular}{cccc}
\hline Grids & Number of elements & Displacement $(\mathrm{m})$ & Diff \% \\
\hline Grid 1 & 254186 & 0.0002819 & - \\
Grid 2 & 279638 & 0.0003267 & 15.89 \\
Grid 3 & 309520 & 0.0003476 & 6.39 \\
Grid 4 & 327648 & 0.0003485 & 0.26 \\
\hline
\end{tabular}

\subsection{Model dimensions}

To minimize the dimensions of the ground model and reduce the analysis time without affecting the model output, the diameter $(d)$ and height $(h)$ of the model are tested within ranges of $5 \sim 8 \mathrm{~m}$ and $2 \sim 5 \mathrm{~m}$, respectively (Fig.3). To test the diameter, the height is fixed at $3 \mathrm{~m}$, and for testing the height, the diameter is fixed at $6 \mathrm{~m}$. The displacements of the same node at the interface of different model dimensions at two-time points are compared as shown in Fig. 4. It can be concluded that the diameter of the ground model has more influence on the calculation results than the height, and the final selected size of the soil model should be at least $7 \mathrm{~m} \times 4$ m. 


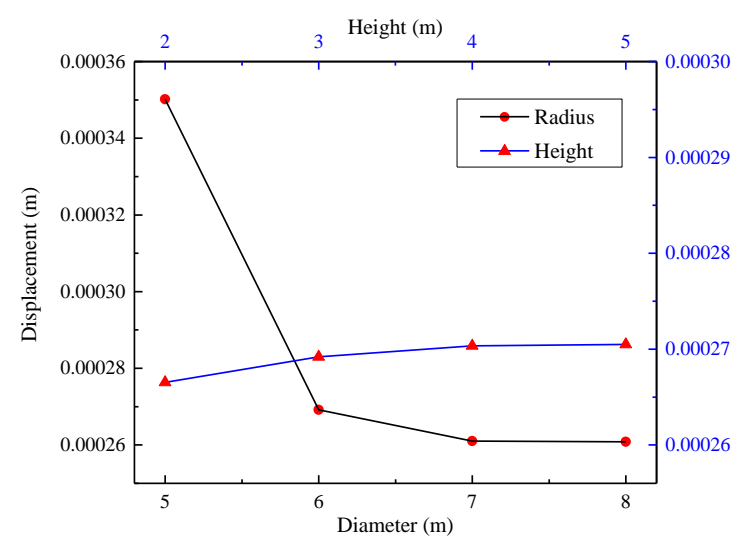

Figure 4. Effects of model dimensions on the displacements.

\subsection{The baseplates-soil details}

The baseplates are treated as a linear elastic material and, as previously mentioned, the soil is modelled using the well-established Drucker-Prager criterion. The plastic behavior of soil is mainly defined by cohesion, internal friction angle and dilation angle. The dilation angle determines the amount of volumetric plastic volumetric strain of the soil and is assumed constant during plastic yielding. If the dilation is equal to the friction, the soil is assumed to follow and associated flow rule, and otherwise it will be non-associative. The material parameters of vibrator and soil are shown in Table.1 [32]. As said before, the interaction between the baseplates and soil is defined by using the master-slave kinematic contact algorithm. The outward surfaces of the baseplates are selected as the master surface while the region containing soil nodes is chosen as the slave surface. The frictional coefficient between the soil and baseplate is 0.4 [37].

Table. 1. Material parameters of soil and vibrator

\begin{tabular}{ccccccc}
\hline & $\begin{array}{c}\text { Density } \\
\left(\mathrm{kg} / \mathrm{m}^{\wedge}\right)\end{array}$ & $\begin{array}{c}\text { Young's modulus } \\
(\mathrm{MPa})\end{array}$ & $\begin{array}{c}\text { Poisson's } \\
\text { ratio }\end{array}$ & $\begin{array}{c}\text { Cohesion } \\
(\mathrm{kPa})\end{array}$ & $\begin{array}{c}\text { Friction angle } \\
\left({ }^{\circ}\right)\end{array}$ & $\begin{array}{c}\text { Dilation angle } \\
\left({ }^{\circ}\right)\end{array}$ \\
\hline Steal & 7850 & $2.12 \times 10^{5}$ & 0.27 & - & - & - \\
Soil & 1889 & 34.0 & 0.43 & 60.57 & 14.08 & 0 \\
\hline
\end{tabular}

\subsection{Boundary conditions}

In the present study, the nodes at the bottom surface are restrained in all three directions . To apply the absorbing boundary conditions, 3D solid continuum infinite elements are employed in this model in the lateral borders. It is noted that though they are called 'infinite elements', they actually refer to boundary conditions whereby the damping is introduced at the finite boundary, so as to minimize the reflected wave energy. To validate the effectiveness of 
the absorbing boundary, the case of an infinite elastic soil column subjected to a vertical force in [38] is studied, which consists of the boundary generated by infinite elements. The material parameters of soil are $\rho=98 \mathrm{MPa}, v=0.001$. The numerical results (both absorbing boundary and fixed boundary) of the vertical displacement suffered by a point $1 \mathrm{~m}$ below the surface in the column are compared. Fig. 5 shows that the result obtained by using infinite elements agrees well with the exact analytical solution. It can be seen here how, in the case of fixed boundaries, there is reflection of waves which is represented by a cyclic vertical oscillation of the top of the soil column, while this is not observed in case of the absorbing boundary. This approach will be implemented in the model developed in this research and the infinite elements are applied on the lateral surface of the ground column.

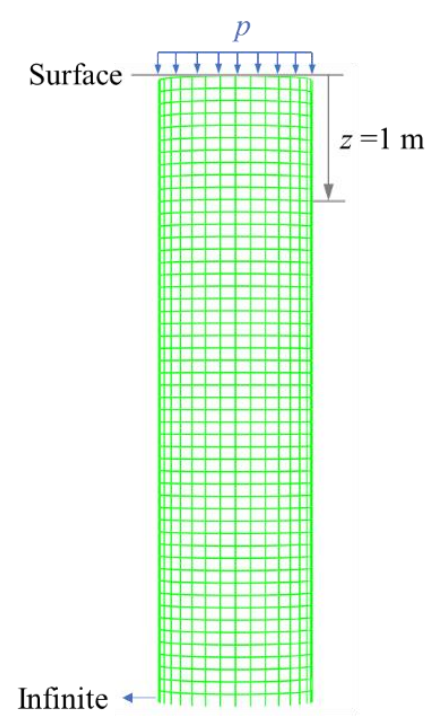

(a)

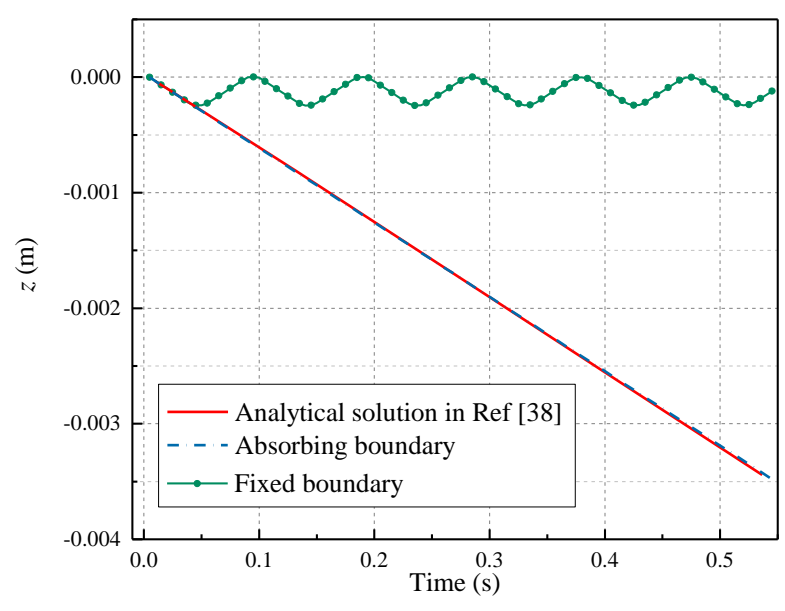

(b)

Figure 5. Validation of the boundary conditions: (a) validation model; (b) comparison between the analytical solution and numerical results, with and without absorbing boundary.

\subsection{Analysis scheme and validation}

The finite element analyses are conducted in several calculation stages. In the first step, the in-situ stresses are initialized in the soil elements only by the application of gravity load. Although in the reality the relative density and stress in the soil column are affected by the installation process of the baseplate, herein it is assumed that these installation effects are of minor importance, and therefore a "wished-in-place" procedure as described was used in the simulation and the baseplate is considered to be in a stress-free state at the beginning of the analysis.

In the following steps, the static analysis is applied to generate static pressure considering the hold-down load. The smooth amplitude curve is employed to improve the contact 
convergence for this static step. After this, a time-varying cyclic load is added on the piston in the dynamic step. For the nonlinear dynamic analysis, the step-by-step Hilber-Hughes-Taylor time integration scheme is employed to calculate the transient response in the time domain. The excitation load of the vibrator is a theoretical prescribed frequency-modulated signal (sweep signal) ranging from $5 \sim 100 \mathrm{~Hz}$. In this section, the frequency of the dynamic load is chosen as $f=50 \mathrm{~Hz}$ and the response of the vibrator at other frequencies will be discussed later in this paper. In the field condition, the excitation frequency varies with time, so the vibrator will not operate at one frequency for a long time.

It is worth to mention here that the purpose of this study is to obtain the response of the vibrator rather than the progressive failure of soil. In previous finite element models for $\mathrm{p}$-wave vibrators, 5 cycles of load were applied to obtain the steady-state response [21]. In the present study, to achieve as overview for comparison purposes and balance the computational effort, 10 cycles are applied. In general, a maximum increment versus period ratio $\Delta t<f / 10$ is a good rule of thumb for obtaining reliable results [39]. Thus, the time step is chosen equal to $0.001 \mathrm{~s}$ and the total number of increments is 200. Figure 6 shows the load locations and amplitude curves of the finite element model.

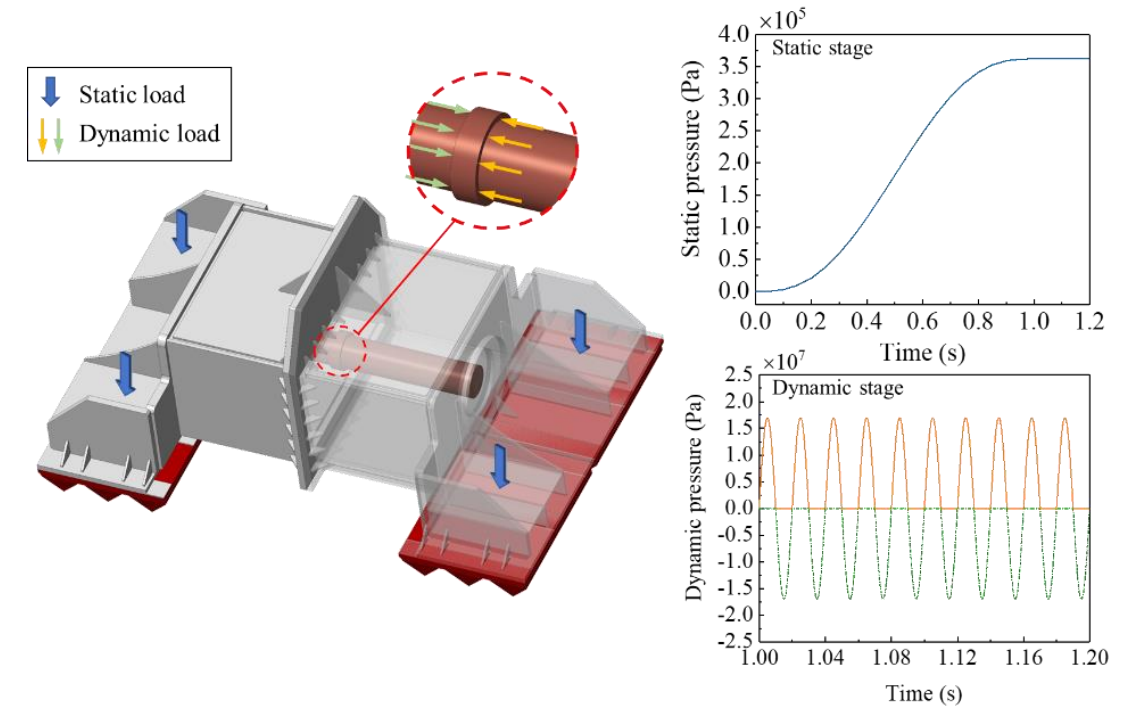

Figure 6. Load locations and the applied loading curves.

The numerical model to simulate the response of the vibrator-ground system should be validated first to ensure the accuracy of the numerical results. To validate the analysis scheme, the velocity of the shear stress obtained by a finite element model of the vibrator-ground system with elastic soil is compared with the velocity calculated by theoretical solution, given by:

$$
V_{s}=\sqrt{G / \rho}
$$

where $G$ is the shear modulus. 
The theoretical solution of the shear wave velocity is $79.33 \mathrm{~m} / \mathrm{s}$. The distance between the $\mathrm{C} 3$ point and $\mathrm{C} 4$ point is $3.6 \mathrm{~m}$ (in Fig. 3 (c)), and the time for the shear tress transferred from $\mathrm{C} 3$ to $\mathrm{C} 4$ is $0.0453 \mathrm{~s}$, hence the numerical result of the velocity is $77.47 \mathrm{~m} / \mathrm{s}$. The difference between the numerical results and the theoretical solution is $2.34 \%$. It approves the reasonability of the numerical simulation model.

\section{Simulation results and discussion}

\subsection{Stress distribution and deformation at the interfaces}

According to the geometry of the vibrator, the location of the dynamic load, which is applied by the piston, is not aligned with the reaction force generated by the ground. This generates a moment and could eventually make the rotation of the vibrator around the $x$-axis (as depicted in Fig.3). The time history of the rotation is represented in Fig. 7. The rotation angle of the vibrator reaches the maximum value in the transient response phase, and then the vibrator enters to the steady-state response, in which the peak absolute value is $1.16 \times 10^{-4} \mathrm{rad}$. Due to the cyclic rotation, the baseplates of the vibrator will be tilted up and down cyclically which can bring about the ununiform contact at the interfaces.

To study the influence of the rotation on the stress distribution at the interfaces, the equivalent pressure stress distribution of the pits generated by the baseplates is also represented in Fig. 7. At the beginning of the dynamic load, the high-stress zone appears on the bottom of the pits and the stress is symmetrical on the right and left interfaces. When the rotation angle reaches the maximum value (the vibrator moving to the left), the right side of the vibrator is tilted up and the minimum stress appears on the interface of the right baseplate. And the stress on the interface of the right baseplate is not equal to that of the left baseplate. Similarly, when the rotation angle reaches the minimum value (the vibrator moving to the right), the lowest stress zone appears on the interface of the left baseplate. In the steady-state response phase, there is also a difference between the stress of the right interface and the left interface, but the difference decreases due to the decrease of the vibrator rotation. When the vibrator is in the equilibrium position, the difference reaches the minimum value, but the stress distribution is still not uniform due to the deformation at the interfaces. 


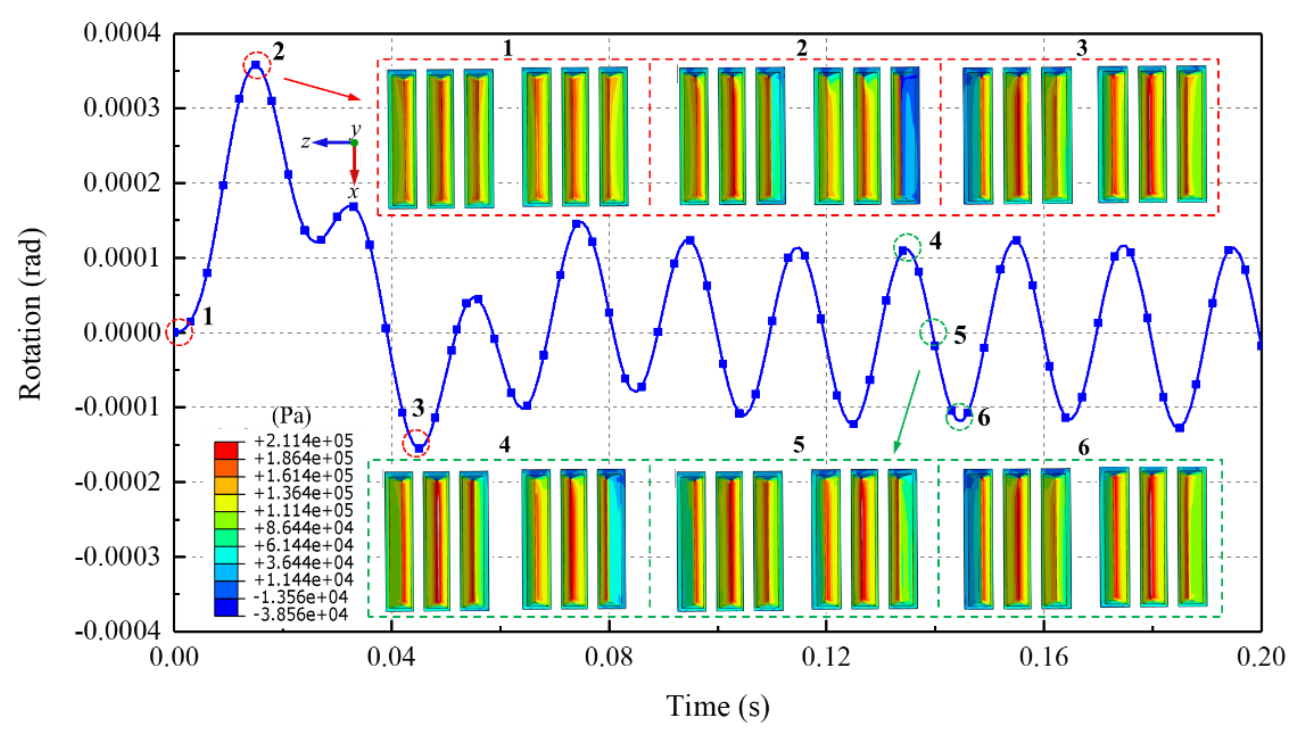

Figure 7. Rotation curves of the vibrator and pressure stress distribution at the interface.

To study the behavior at the interfaces, the displacement curves of the reference points (according to Fig. 3(b)) at pits of the left baseplate are shown in Fig. 8. The displacements for the reference points in the vertical direction $(y)$ in the soil face of the contact, due to the rotation of the vibrator, are represented in the figure. The vertical displacements at the lateral points, A1 and $\mathrm{C} 3$, are smaller than the other reference locations, and the maximum values appear on the points at the bottom of the interfaces (A2, B2, and C2). All the displacement curves of these points offset in the vertical direction and the average values increase with the loading time. The average of the displacement curves represents that the pits on the ground become deeper due to the dynamic load, as shown in the plot of contours. Fig. 9(b) shows the horizontal displacement (z-direction) of the reference points. The maximum values of the horizontal displacement appear on the points $\mathrm{A} 1$ and $\mathrm{C} 3$, and the differences of the displacement of the other points are very small. It is also seen that the displacement curves of A1 and C3 average values in the horizontal direction, and the mean values increase with the loading time. Thus, the area between the curves of $\mathrm{A} 1$ and $\mathrm{A} 2$ as well as the curves of $\mathrm{C} 1$ and $\mathrm{C} 3$ increases, which means the size of pit $\mathrm{A}$ and pit $\mathrm{C}$ increases, as shown in the plot of contours. The size of the middle pit $\mathrm{B}$ basically maintains unchanged. According to Fig. 8, the deformation of the interfaces between the vibrator and ground are ununiform in both vertical and horizontal direction, which is due to the uneven contact and affects the stress distribution. 


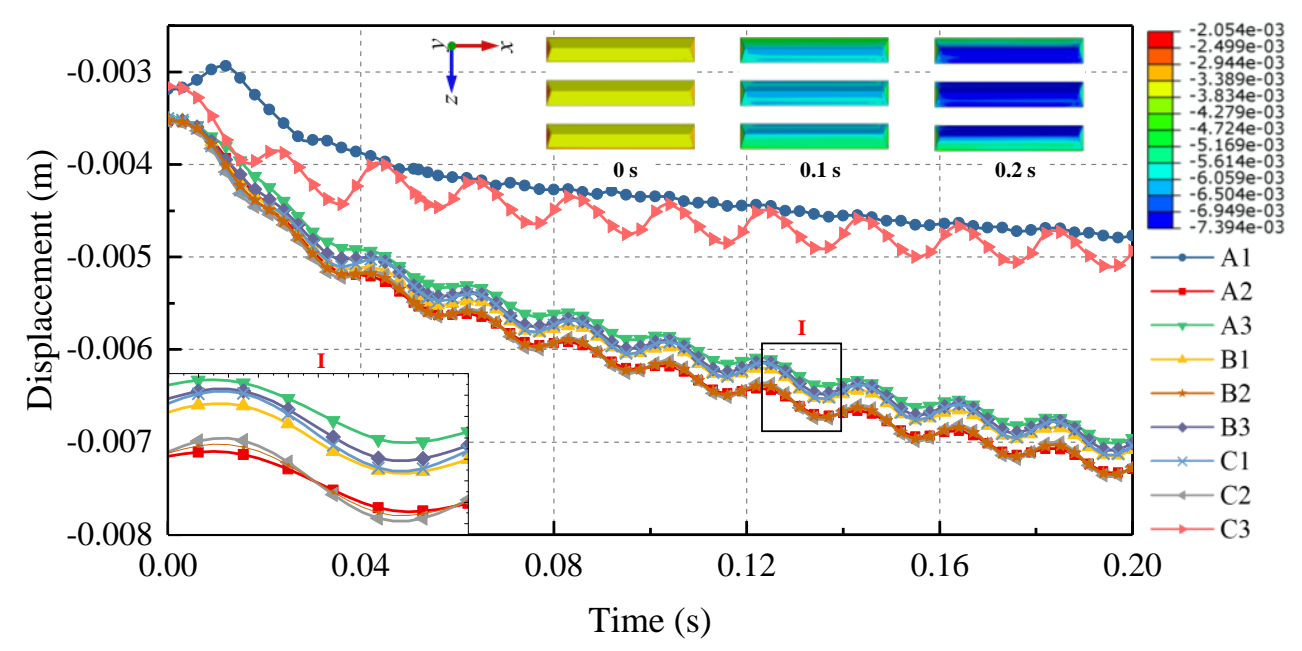

(a)

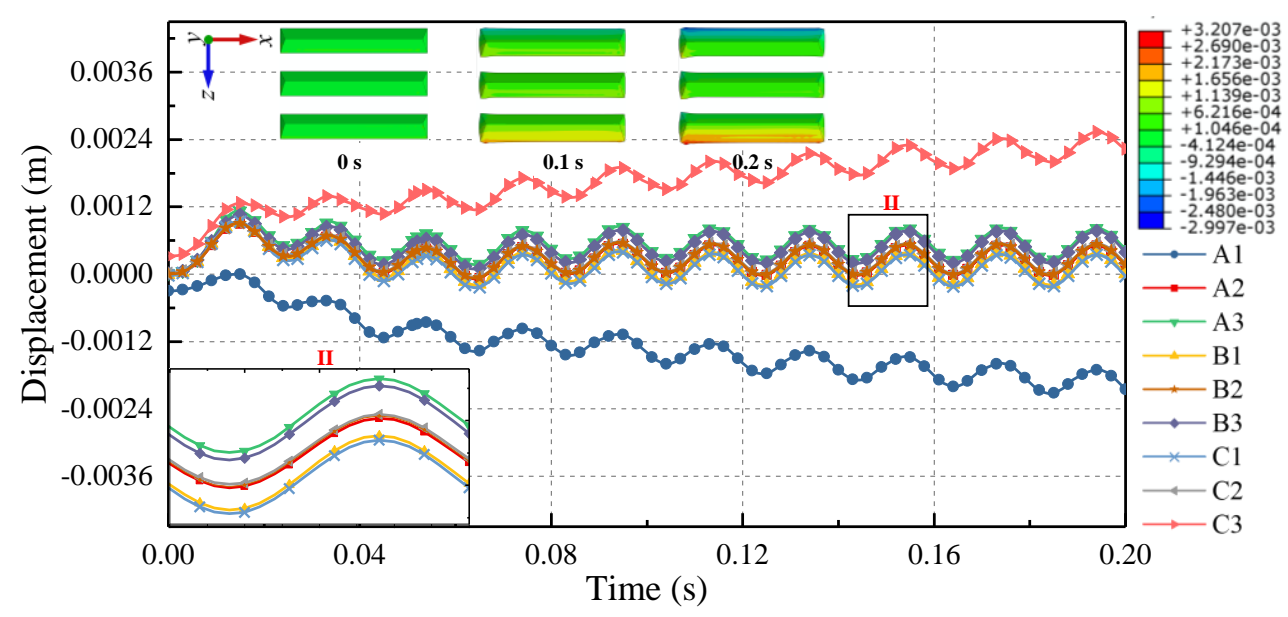

(b)

Figure 8. Displacement of the reference points in different direction at the interface: (a) vertical direction $-y$; (b) horizontal direction $-z$.

The average values of the displacement curves of the reference points come from the plastic deformation of the ground. The contours of the equivalent plastic strain of the ground are shown in Fig. 9. At the beginning of the dynamic loading phase, the high plastic strain appears on the ends of the pits, and then it extends to the whole contact interfaces when the loading cycles increase. It can be seen that the values of equivalent plastic strain increase with the dynamic loading cycles, as well as the size of the pits. The maximum value of the equivalent plastic strain appears on the inner sides of the pits. 


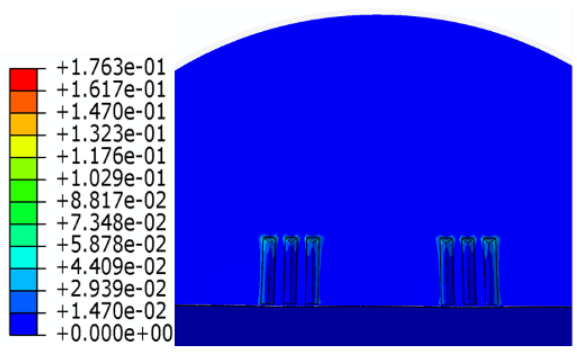

0 cycle

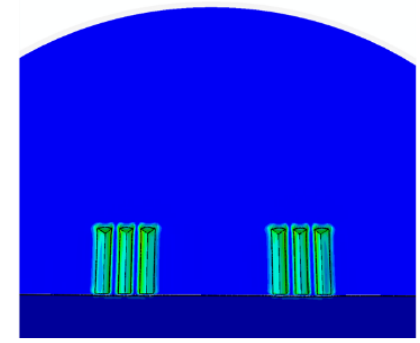

5 cycles

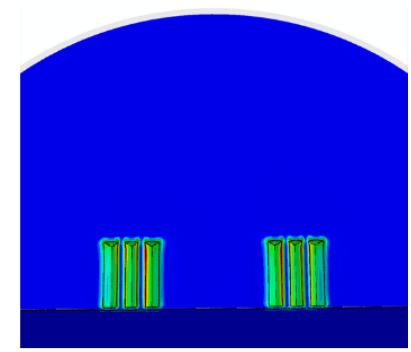

10 cycles

Figure 9. Contours of equivalent plastic strain (deformation scale factor $=10)$.

\subsection{Propagation of the seismic wave}

In order to figure out the propagation of the seismic wave generated by the s-wave vibrator, the plots of the contours of the shear stress of the ground, varying with time in one loading cycle, are shown in Fig. 10. The high and low shear stress zones appear on both sides of the interface respectively. The high and low stresses are transmitted to the deeper earth alternatively. In the $x-y$ plane, the propagation of the 3D outgoing wave can be observed. It can also be seen how the seismic wave is absorbed when it arrives at the boundary, which proves the effectiveness of the absorbing boundary.

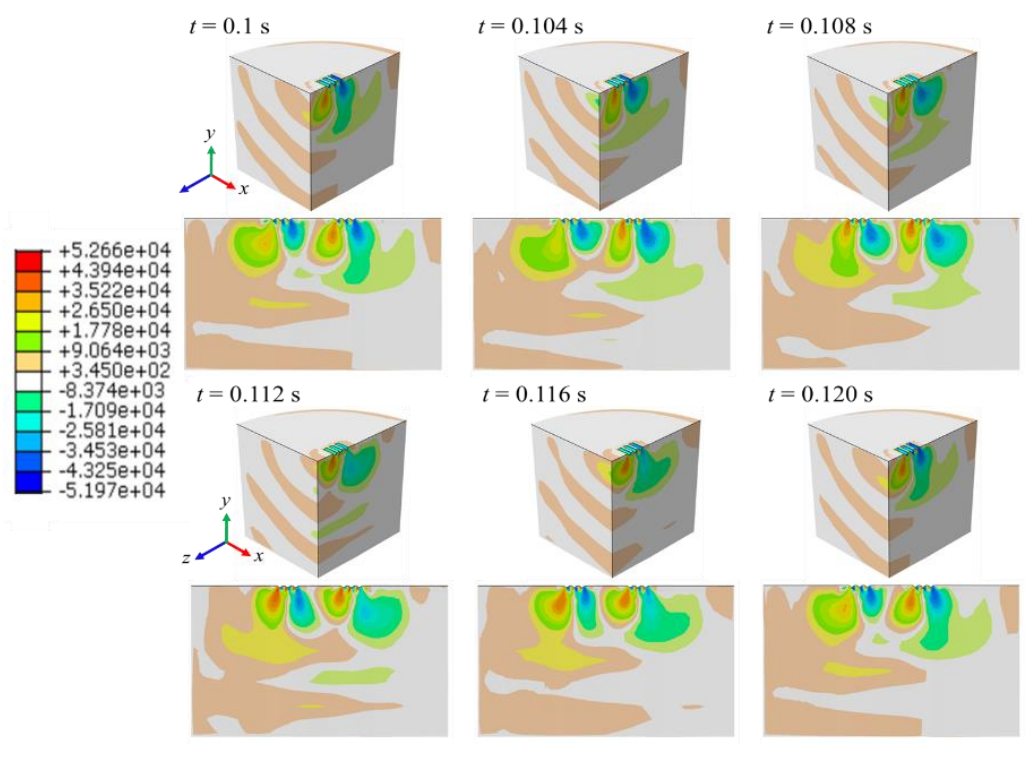

Figure 10. Shear stress of the ground varies with time.

Figure 11 shows the time-history curves and amplitude-frequency curves of the shear stress at the reference elements (according to Fig. 3(c)). The initial stress generated by the weight of soil is set to zero to define the datum level for static and dynamic loading phase. Due to the plastic deformation of the pits, the peak of the shear stress curves at point $\mathrm{C} 3$ of which the depth is $0 \mathrm{~m}$ is a little flat. The amplitude of the shear stress decreases with the increase of the depth of points. It is also seen that both subharmonics and higher harmonics exist in the 
excitation signal, and the higher harmonics dissipates in the propagation process. When the depth is equal to $2.6 \mathrm{~m}$, the higher harmonics have little influence on the shear stress.
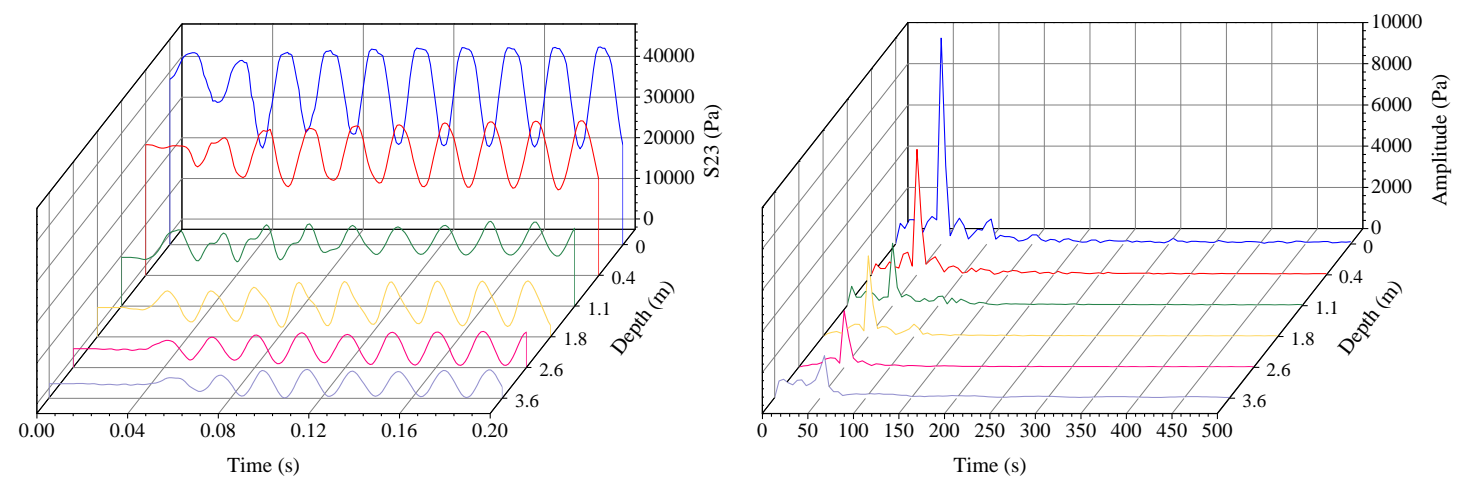

Figure 11. Shear stress at the reference points: (a) time-history curves; (b) amplitudefrequency curves.

\subsection{Ground force and energy transfer}

The ground force emitted by the vibrator is one of the most important performance indexes, as a larger ground force provides a deeper exploration depth. In the present study, the ground force is assigned as the horizontal reaction force between the vibrator and ground, as shown in Fig. 12. The amplitude of the ground force in the horizontal direction of the s-wave vibrator is about $35000 \mathrm{~N}$. According to the distribution of the contact pressure at the interfaces, the minimum value of the contact pressure is zero, which means that there is a loss of contact at some area of the interfaces. The area of contact loss appears at the end of the pits. When the vibrator is at the peak point $\left(T_{1}\right)$, the low-pressure zone appears on the interface of the left baseplate and the minimum value is $2.68 \times 10^{2} \mathrm{~Pa}$. When the vibrator reaches pint $\mathrm{T}_{3}$, the lowpressure zone transmits to the right interface. The deformation of the interfaces also has effects on the ununiform contact distribution even when the vibrator is at the equilibrium position $\left(\mathrm{T}_{2}\right)$. 


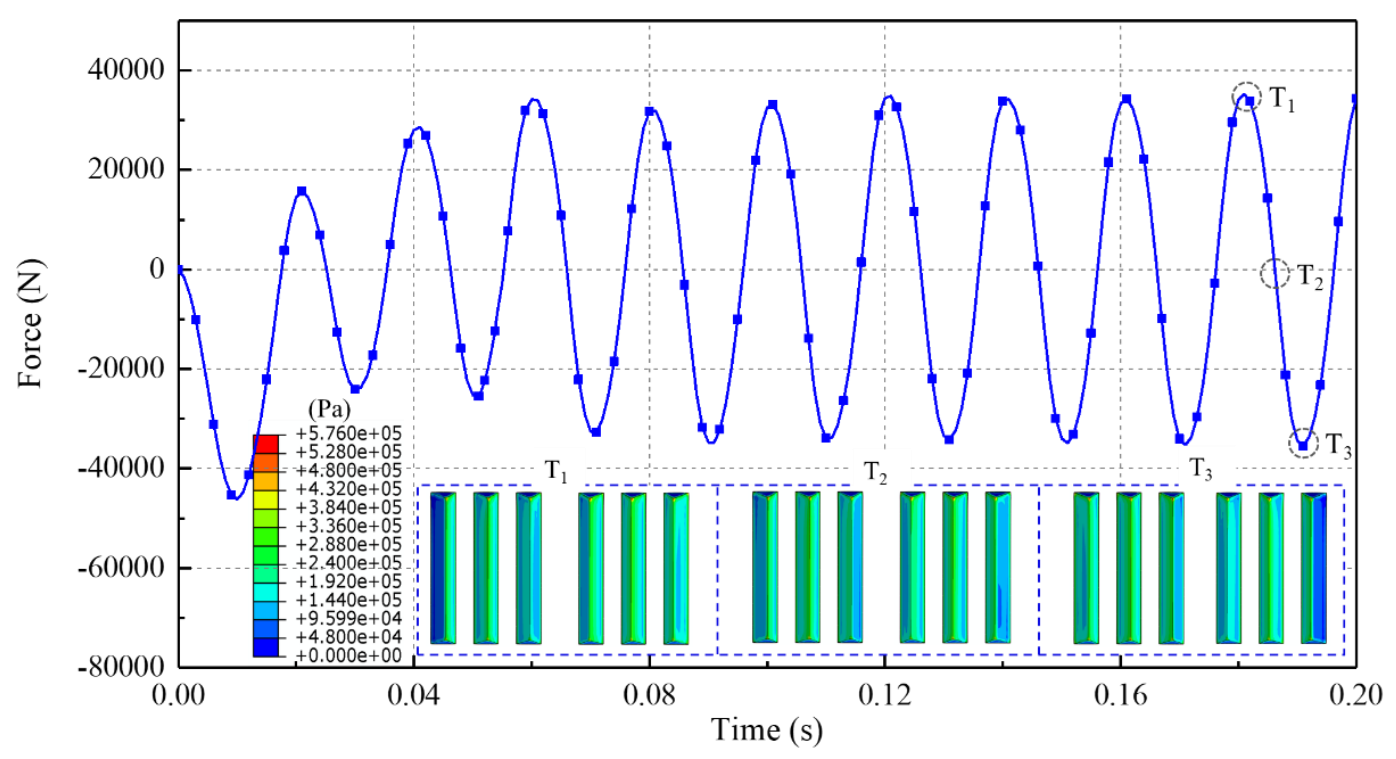

Figure 12. Ground force of the vibrator and distribution ofcontact pressure.

One of the advantages of the seismic vibrator is that it can control the excited seismic energy and accumulate energy through increasing the vibration time. Figure 13 shows the characteristics of the energy transferred by the vibrator-ground system in the dynamic loading phase. To investigate the that, the initial energy generated by the static load is set as zero. It is seen that the external work increases with the loading time. Meanwhile, the energy dissipation due to the plastic deformation and friction also increases with time, but the energy dissipated by friction is much less than that dissipated by plasticity. The effective transferred energy is the real energy of the ground obtained from the vibrator, and the larger the effective energy the deeper the exploration depth. In Fig. 15(a), the effective transferred energy decreases slightly and then increases with the loading time. It means the ground will release some strain energy at the beginning of the dynamic loading phase and then obtain energy from the vibrator. Figure 13 shows that most of the external work is dissipated by the plastic deformation and the effective transferred energy is quite small. The ratio of the effective transferred energy in the external work increases dramatically then decreases for a period, and finally remains stable. The minimum value of the transferred ratio is $8.94 \%$. Thus, although increasing the loading time can increase the effective transferred energy, the transferred ratio is small and barely change, which demonstrates that the selected number of cycles for this analysis represents the steady solution and can be used for comparison purposes. 

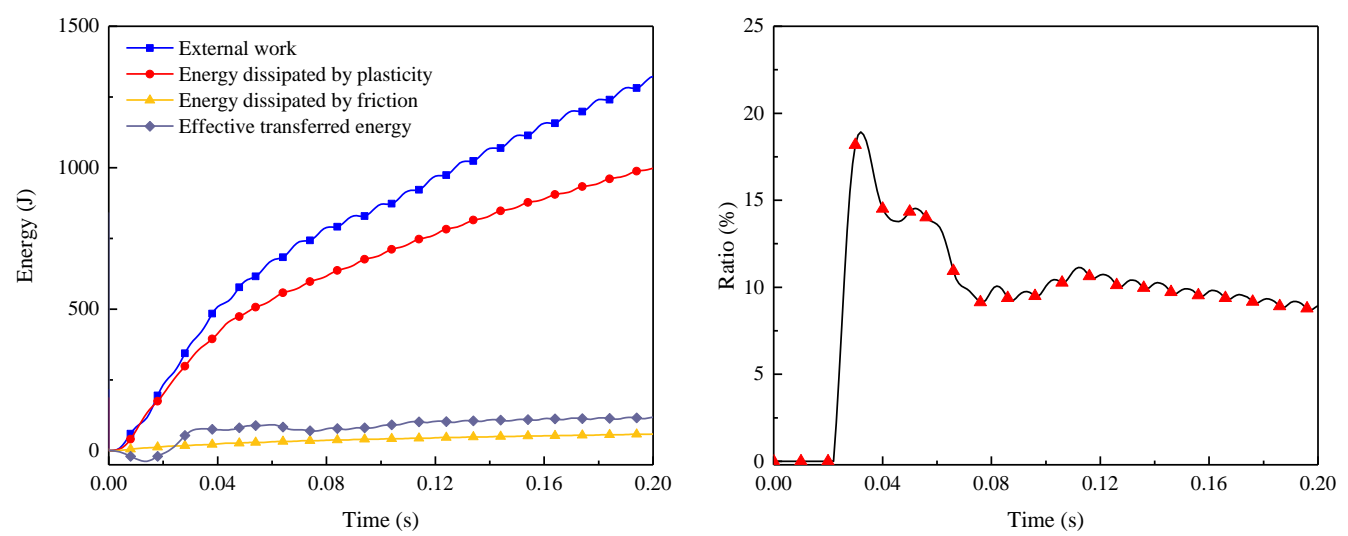

Figure 13. Energy characteristics of the vibrator-ground system: (a) energy of the ground; (b) energy transfer ratio.

\subsection{Evaluation criterion of the vibrator performance}

An evaluation criterion can be developed from the above analysis to evaluate the performance of the s-wave vibrator for different system parameters. The plastic deformation and shear stress within the soil domain are used to evaluate the accuracy of the vibrator. Meanwhile, the output force and the energy characteristics are employed to quantify the power of the vibrator.

\section{Parametric analysis of the system}

A series of finite-element analyses are carried out to study the influence of the system parameters on the dynamic response of the vibrator-ground system. Several factors are considered in this parametric study namely, (i) the excitation frequency (ii) the soil properties (iii) the embedded depth of the baseplates. The results obtained from this investigation with reference to various parameters are presented next. The parameters for the parametric studies are shown in Table. 2.

Table. 2 The system parameters for different cases

\begin{tabular}{cccc}
\hline & Excitation frequency $(\mathrm{Hz})$ & Soil properties & Embedded depth $(\mathrm{mm})$ \\
\hline Case I & $5 \sim 100$ & Clay & 69 \\
Case II & 50 & Soil, Clay, Elastic & 69 \\
Case III & 50 & Clay & $33 \sim 99$ \\
\hline
\end{tabular}

\subsection{Case I: effects of excitation frequency}

Due to the sweep excitation for the vibrator, it is necessary to study the vibrator 
performance under different frequencies. The normal range of the excitation frequency for swave vibrators is $5 \sim 100 \mathrm{~Hz}$, and therefore the following frequencies are chosen to study the influence of this parameter: $5 \mathrm{~Hz}, 10 \mathrm{~Hz}, 30 \mathrm{~Hz}, 50 \mathrm{~Hz}, 75 \mathrm{~Hz}$, and $100 \mathrm{~Hz}$.

In order to fully understand the effect of the different input frequencies, it is first necessary to determine the natural frequency of this system. Modal analysis is an efficient way to determine the natural frequency and the mode shape of a system, but is based on the fact that the vibration response of a linear time-invariant dynamic system can be expressed as the linear combination of a set of the natural modes of vibration, and it cannot take into account nonlinear factors, as the plastic behavior of the soil or the frictional nature of the soil-baseplates interface. Thus, in the present study, the natural frequency of the system in the horizontal direction is carried out by using a full dynamic, free vibration analysis, as shown in Fig. 14. We applied a horizontal displacement on the vibrator and removed it suddenly. After some free oscillations, the vibrator will stay at the equilibrium position. Fig. 14(b) shows that the cycle of the free vibration is about $0.08 \mathrm{~s}$, so the natural frequency is $12.5 \mathrm{~Hz}$.

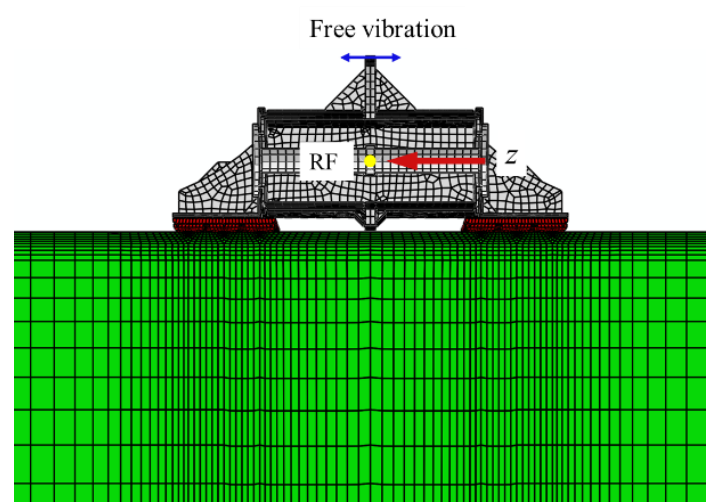

(a)

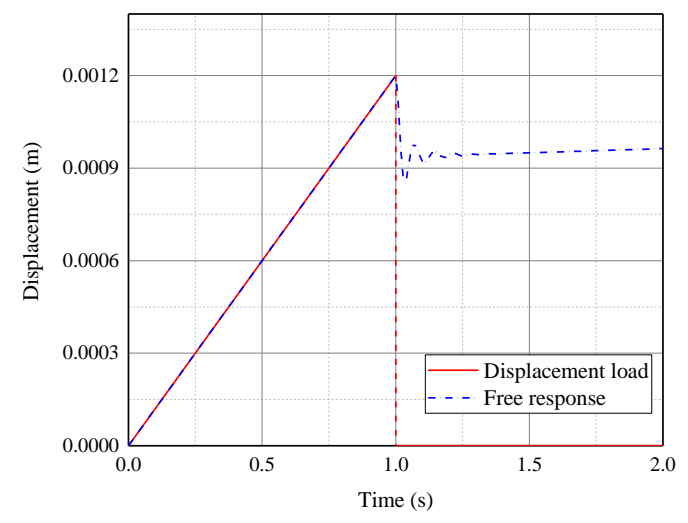

(b)

Figure 14. Free vibration analysis: (a) simulation model; (b) load and response curves.

The equivalent plastic strain of the ground at the end of the 10 cycles of dynamic loading phase, for the different frequencies, is shown in Fig. 15. It is seen that the plastic deformation and the size of the pits increase with the excitation frequency lower than $10 \mathrm{~Hz}$, and then decrease with the increase of the frequency. Generally, the vibrator uses a linear increasing sweep signal, the baseplates can generate larger plastic deformation in the low-frequency phase and result in bigger pits. However, due to the decrease of the displacement with the excitation frequency, the possibility of the loss of contact will increase which can reduce the accuracy of the high-frequency output signal. 


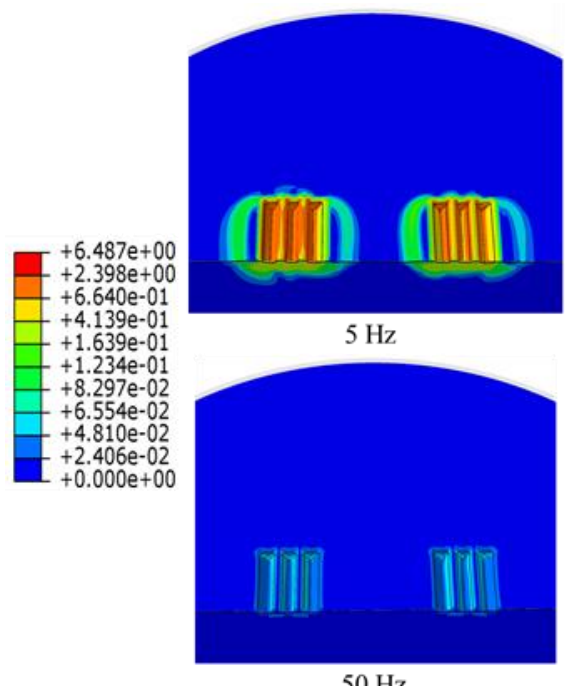

$50 \mathrm{~Hz}$

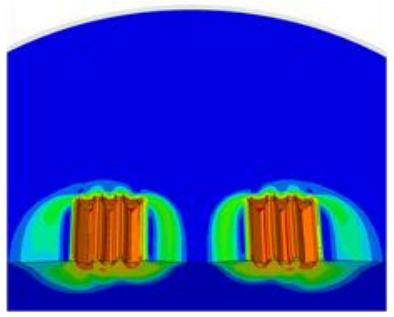

$10 \mathrm{~Hz}$

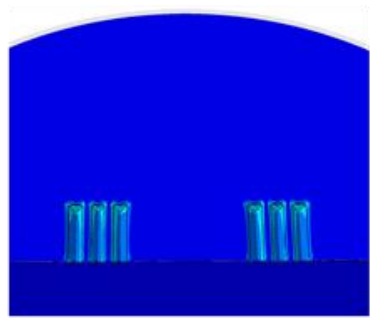

$75 \mathrm{~Hz}$

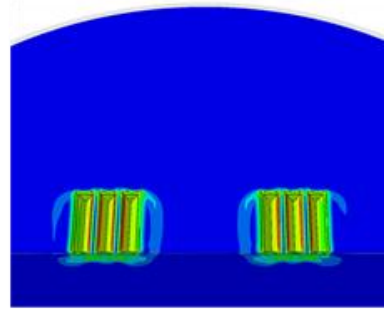

$30 \mathrm{~Hz}$

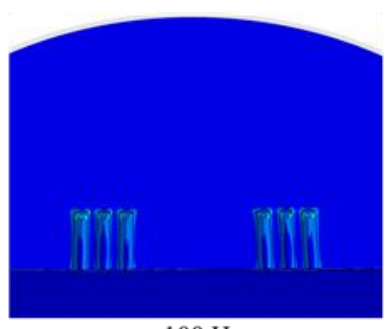

$100 \mathrm{~Hz}$

Figure 15. The equivalent plastic strain for different frequency.

Figure 16 shows the shear stress in the soil side of the interface of the reference point (A1) for different excitation frequencies. It can be seen that the shear stress at the surface reference point decreases with the increase of the excitation frequency, however, there is also a more serious distortion of the signal in the low-frequency phase. When the excitation frequency is 5 $\mathrm{Hz}$, there is no trough in the stress curve and the reason is that A1 is at the interface between the baseplates and ground, and there is loss of contact when the vibrator moves to the opposite direction. For the shear stress curves of $5 \mathrm{~Hz}$ and $10 \mathrm{~Hz}$, it is also seen that there is a decline in the peak of the stress curves in the last three loading cycles. Due to the plastic deformation, the size of the pits increases, and the loss of contact can be observed even when the vibrator moves to the surface of $\mathrm{C} 3$. The distribution of the contact pressure at the interface near $\mathrm{C} 3$ at the last four peak point for $5 \mathrm{~Hz}$ is shown in Fig. 16, and it is inferred that the contact pressure of $\mathrm{C} 3$ decreases in the last four loading cycles which brings about the decrease of the peak. For 30 $\mathrm{Hz}$, the peak and trough of the shear stress are insufficient, thus there is also a contact loss at the interfaces. With the increase of the excitation frequency, the distortion of the curves decreases which means the loss of contact disappears at higher frequency and the contact at the interfaces become more uniform. According to the above analysis, a lower excitation frequency is beneficial for generating bigger shear waves, but the harmonic distortions are more serious. Thus, it is necessary to optimize the structures of the s-wave vibrator to improve the accuracy of the generated waves in lower frequency or increase the amplitudes of the generated waves in higher frequency. 


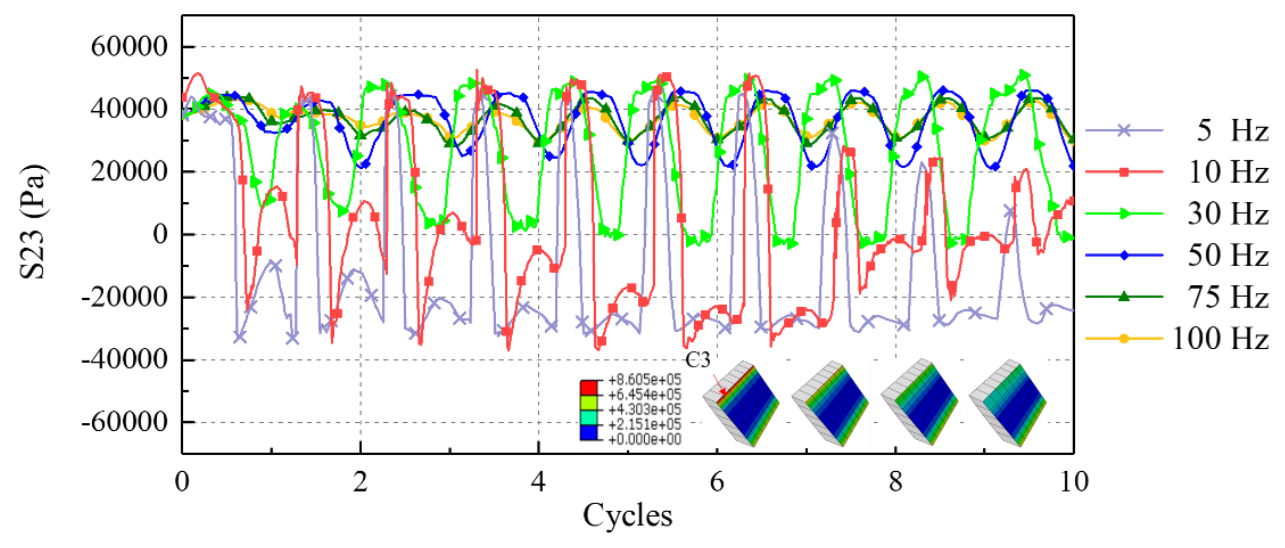

Figure 16. Comparison of the shear stress for different frequency at point $\mathrm{C} 3$.

Figure 17 shows the ground forces of the vibrator for different excitation frequencies. Because $10 \mathrm{~Hz}$ is closer to the natural frequency, the output force of the vibrator for $10 \mathrm{~Hz}$ is larger than for the other frequencies. In general, the vibrator can generate larger outputs in lowfrequency according to Fig. 17. However, the distortion of the curves is also more serious in low-frequency phase due to the larger plastic deformation, as previously discussed. The dimensionless frequency is given as $\Omega=f_{e} / f_{n}$, where $f_{e}$ is the excitation frequency and $f_{n}$ denotes the natural frequency. Fig. 17(b) shows the variation ground force with the dimensionless frequency. As expected, the peak amplitude occurs at around $\Omega=1$, for which the excitation frequency is equal to the natural frequency. As the excitation frequency increases or decreases from the natural frequency, the amplitude decreases.

The energy characteristics of the system for different frequencies are represented in Fig. 18 When $f_{e}>10 \mathrm{~Hz}$, the external work decreases with the increase of the excitation frequency. At the beginning, the external work for $10 \mathrm{~Hz}$ is smaller than that for $5 \mathrm{~Hz}$ but it exceeds that for $5 \mathrm{~Hz}$ at last five loading cycles. Thus, the vibrator needs larger pump displacement in the low-frequency case to satisfy the demand of external energy. There is also a decrease in the effective transferred energy with the increase of frequency except for $100 \mathrm{~Hz}$. It is seen that the effective transferred energy at $100 \mathrm{~Hz}$ basically equals to that at $75 \mathrm{~Hz}$. Thus, the low-frequency excitation is better for the propagation of the outgoing waves deeper in the soil domain, which can improve the exploration effectiveness. However, due to the increase of the plasticity dissipation, the ratio of the effective transferred energy in the external work decreases significantly with the decrease of the frequency. The average values of the transfer ratio for 5 $\mathrm{Hz}, 10 \mathrm{~Hz}, 30 \mathrm{~Hz}, 50 \mathrm{~Hz}, 75 \mathrm{~Hz}$ and $100 \mathrm{~Hz}$ are 1.23\%, 1.78\%, 6.90\%, 9.69\%, 11.79\%, and $11.91 \%$, respectively, which demonstrates that the most efficient frequencies are $75 \mathrm{~Hz}$ and 100 Hz. 


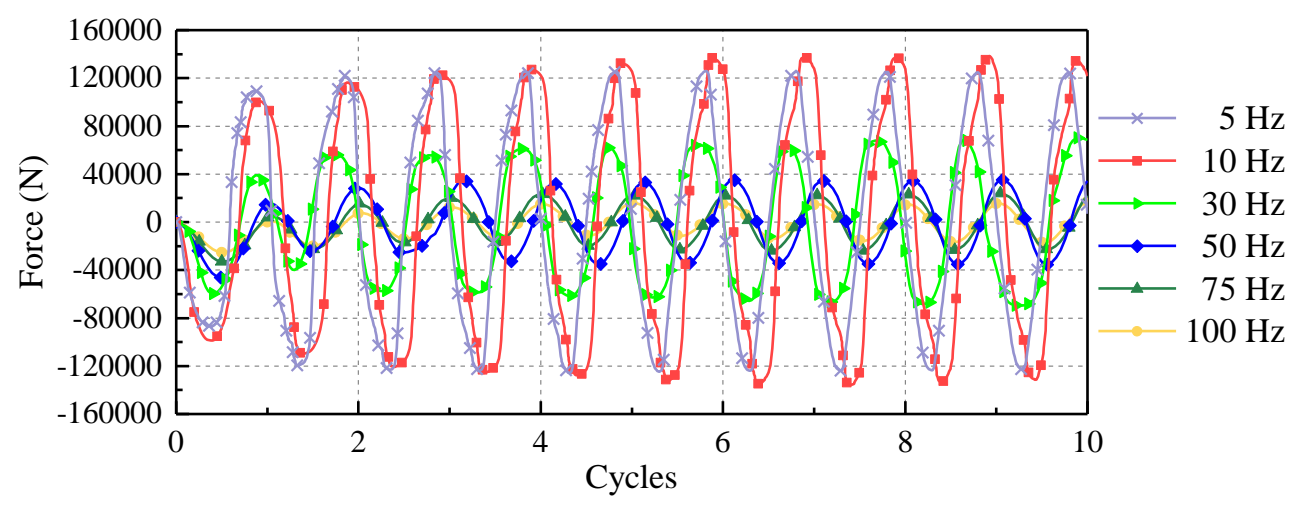

(a)

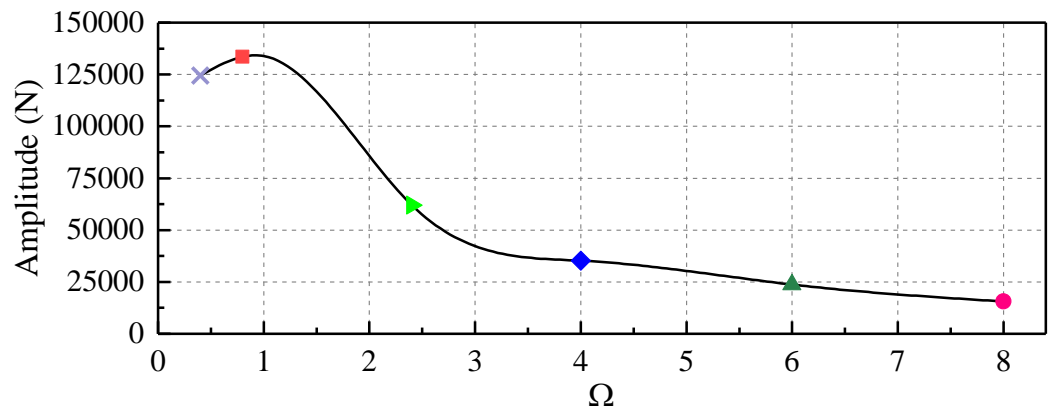

(b)

Figure 17. The ground force of the virator for different frequency: (a) time-histoty curves; (b) $\Omega$-amplitude curves $\left(\Omega=f_{e} / f_{n}\right)$.

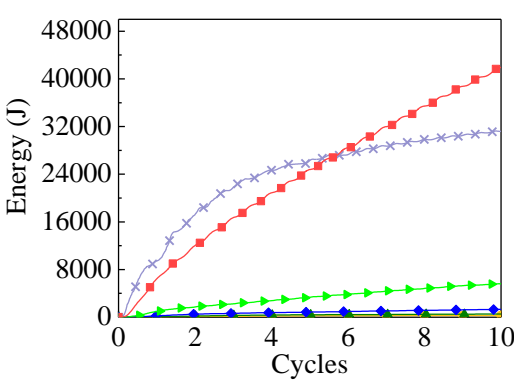

(a)

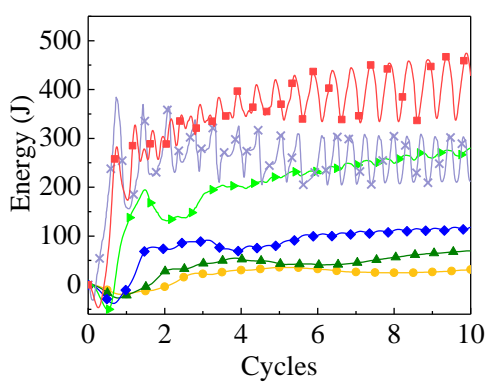

(b)

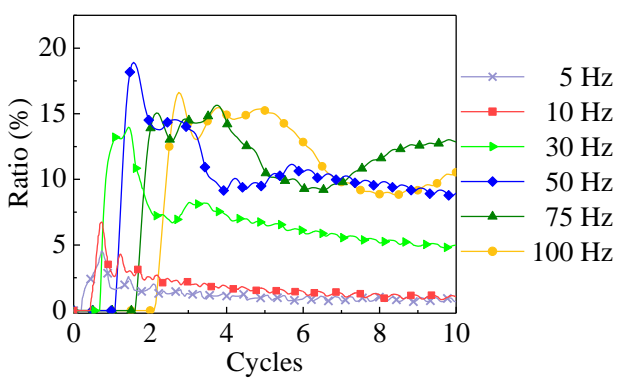

(c)

Figure 18. The energy characteristics for different frequency: (a) the total energy of ground;

(b) the effective transferred energy; (c) the transferred ratio.

\subsection{Case II: effects of soil properties}

The seismic vibrator needs to operate in multiple conditions to meet exploration requirements in different regions. Thus, different soil material in these operation regions can affect the performance of the vibrator. In order to study the influence of the soil materials, the dynamic response of the vibrator-ground system in sand is investigate herein. The following parameters are used for sand: Young's modulus of $20 \mathrm{MPa}$, Poisson's ratio of 0.3 , friction angle 
of $30^{\circ}$, and dilation angle of $5^{\circ}$ [40]. Moreover, an elastic model is also developed to analyze the influence of soil plasticity, and the material parameters are Young's modulus of $34 \mathrm{MPa}$, Poisson's ratio of 0.43 .

Figure 19 compares the equivalent plastic strain of the ground for different soil materials at the end of the dynamic loading phase. The maximum value of the equivalent strain for sand is 6.487 , which is larger than that for clay. It is also seen that the plastic deformation area of ground increases for sand, as well as the size of the pits. The time-history of the shear stress and amplitude-frequency curves at the reference points of the system in clay, sand and elastic soil are shown in Fig. 11, Fig. 20 and Fig. 21, respectively. The amplitudes of the shear stress at the surface point $\mathrm{A} 1$ are 10037.51 Pa for clay, 5392.42 $\mathrm{Pa}$ for sand, and 16369.08 $\mathrm{Pa}$ for elastic soil. At the bottom point of the ground model, the amplitudes of the shear stress are 1974.26 Pa for clay, 1546.25 Pa for sand, and 2536.20 Pa for elastic soil. Moreover, the distortion of the signal in sand is more serious than the other soils, and the higher harmonics still can affect the shear stress curve in the deeper soil. Thus, the operation of vibrator in sand will reduce the strength of the outgoing signal and increase the harmonic distortion.

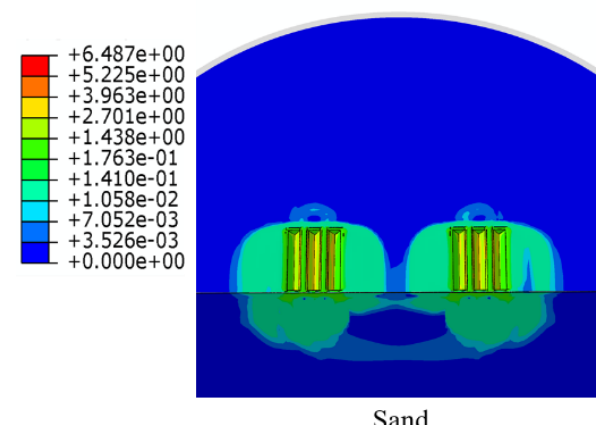

Sand

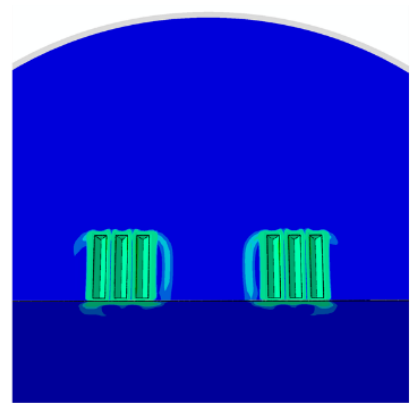

Clay

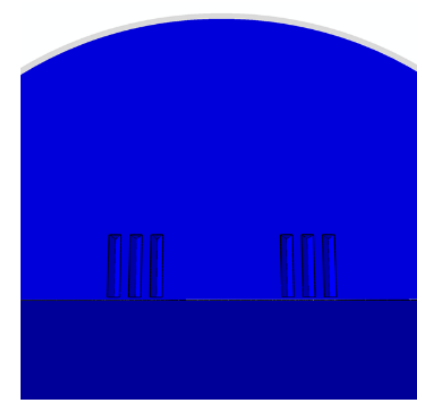

Elastic

Figure 19. Comparison of the plastic deformation for different soils.
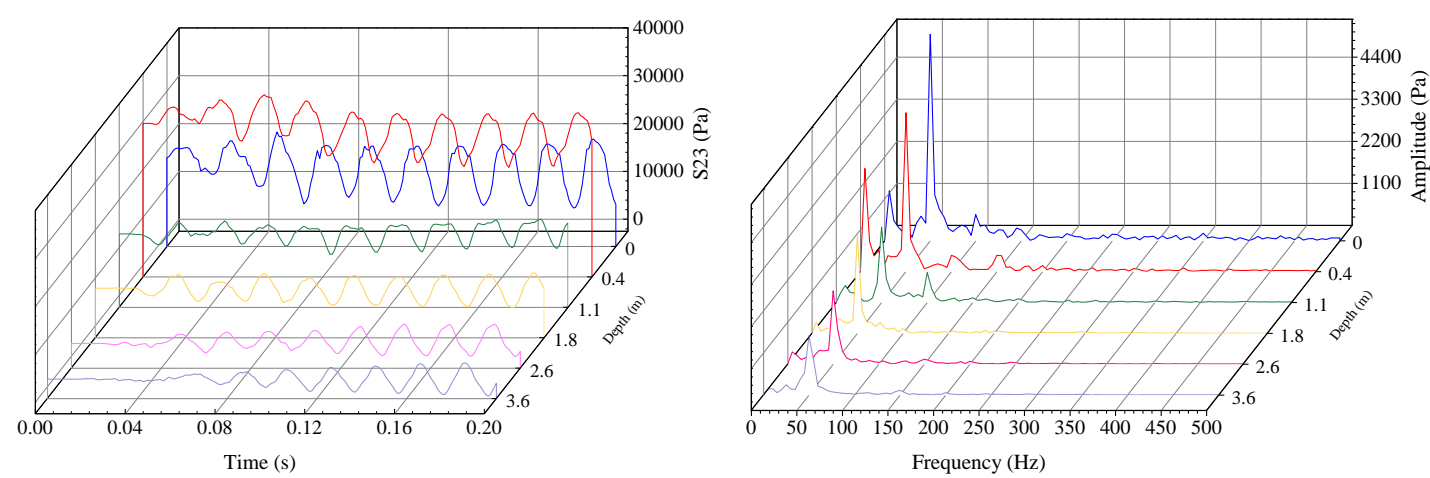

Figure 20. Shear stress at the reference points in sand: (a) time-history curves; (b) amplitudefrequency curves. 

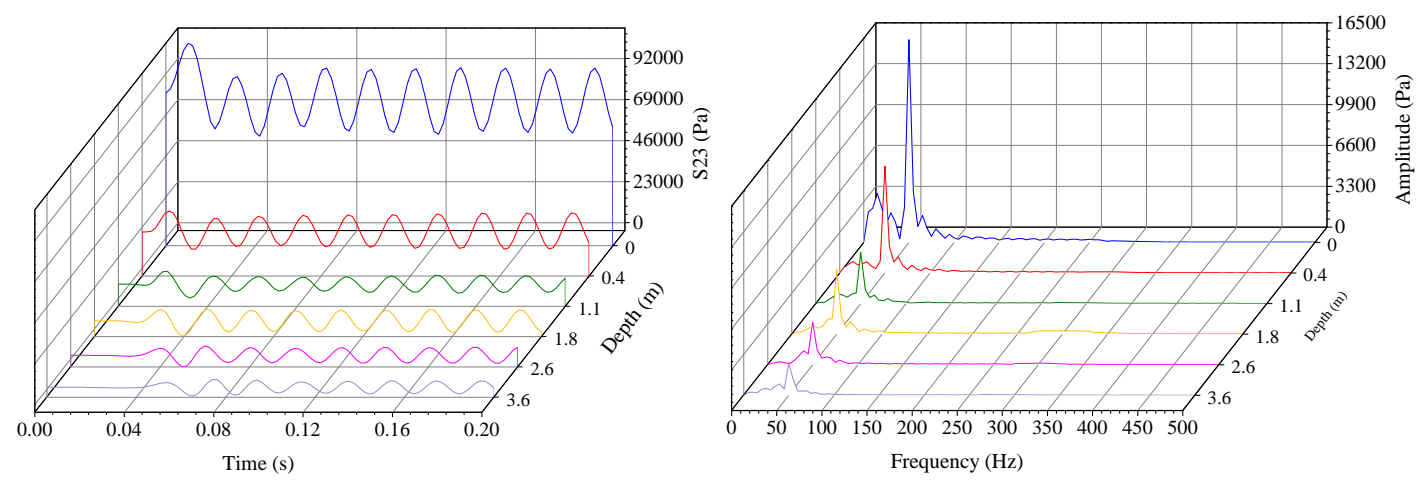

Figure 21. Shear stress at the reference points of system in elastic soil: (a) time-history curves; (b) frequency-amplitude curves.

To evaluate the power of the s-wave vibratorin different soils, the ground force applied by the vibrator is shown in Fig. 22. The ground force of the plastic soil model decreases compared with that of the vibrator in elastic soil. Therefore, it is inferred that the soil plasticity can reduce the powerof the vibrator. The ground force of the vibrator in sand is smaller than it in clay, so the operation condition of sand has negative effects on the vibrator output which can reduce the exploration effectiveness. The plots of the distribution of contact pressure at the same time points $\left(T_{1}, T_{2}, T_{3}\right)$ for elastic soil and sandy soil are also shown. The contact pressure at the interfaces of the elastic model is more uniform even when the vibrator is at peak point or trough point. And the maximum value appears on the corners of the pits on the elastic ground. For the system in sand, the contact at the peak point or trough point is uneven, but the minimum value is larger than zero. Thus, there is no loss of contact at the interfaces for the system in sand, contrarily as observed in the system in clay. But the area of low-pressure zone at the interface in sand is larger than that in clay.

Figure 23 compares the energy characteristics of the system in clay and sand. The external work of the system in sand increases, but there is no significant increase in the effective transferred energy. Therefore, the transfer ratio for the system in sand is smaller than that of the system in clay. It means that the vibrator in sand needs more energy from the hydraulic system but the energy dissipation also increases which can reduce the exploration depth. Therefore, we can conclude that the s-wave vibrators are expected to work better in clayey soils than in sands. 


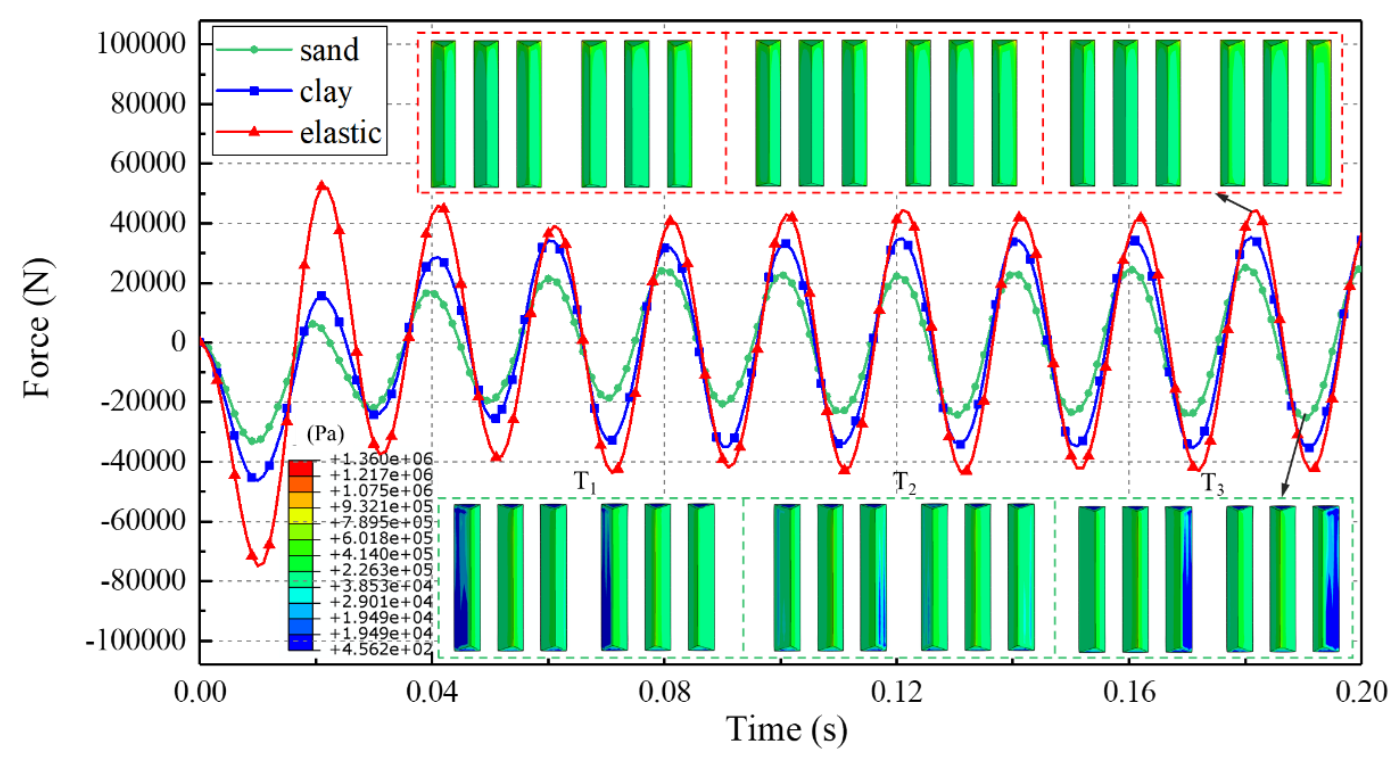

Figure 22. The ground force of the vibrator and the contact pressure distribution at the interface for different soils.

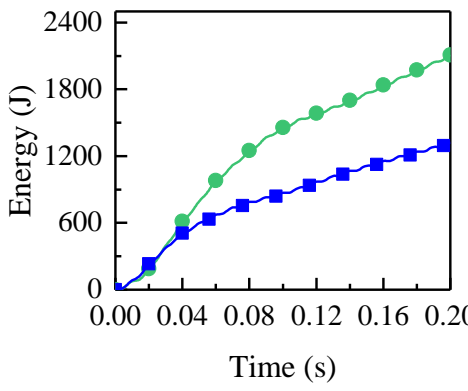

(a)

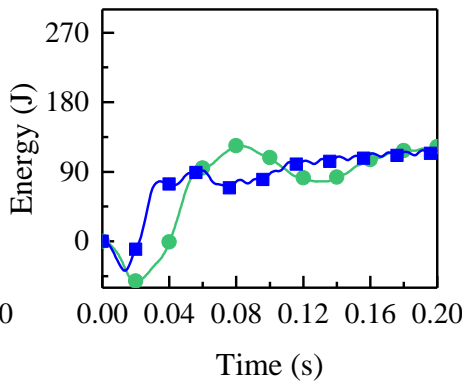

(b)

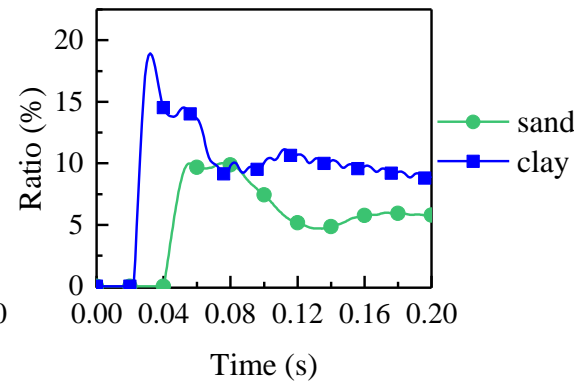

(c)

Figure 23. The transferred energy for different soil material: (a) total energy; (b) effective trandferred energy; (c) the transfer ratio.

\subsection{Case III: effects of embedded depth}

The embedded depth of the baseplates also has influence on the performance of the vibrator and its output signal. To study the influence, a series of finite element models are carried out varying embedded depth of $33 \mathrm{~mm}, 69 \mathrm{~mm}$, and $99 \mathrm{~mm}$ in soil, as shown in Fig. 24.
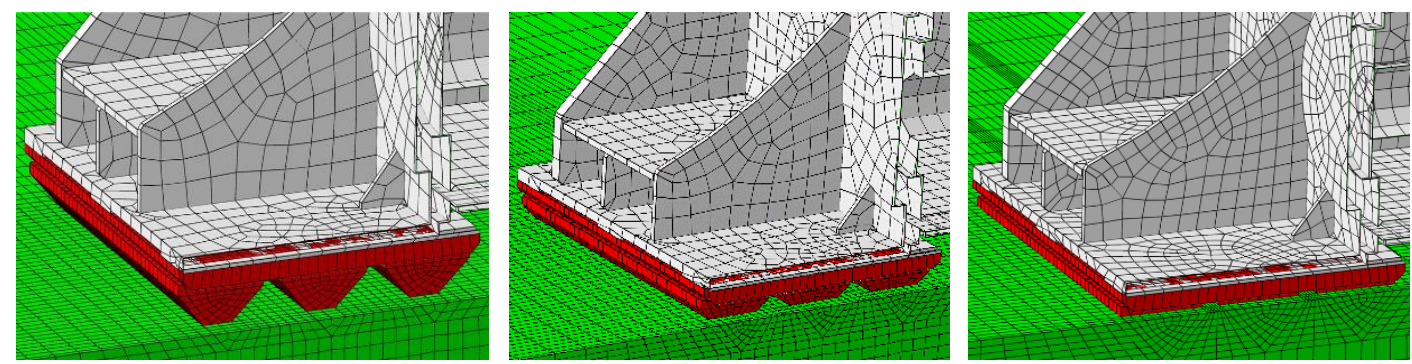
(a)

(b)

(c)

Figure 24. Finite element models of different embedded depth: (a) 33 mm; (b) 69 mm; (c) 99 $\mathrm{mm}$.

Figure 25 shows the equivalent plastic strain of the ground at the end of the dynamic loading phase varying with the embedded depth. The maximum value of the equivalent plastic strain for $33 \mathrm{~mm}, 69 \mathrm{~mm}$, and $99 \mathrm{~mm}$ are $4.02,1.76 \times 10^{-1}$, and $0.066 \times 10^{-2}$, and it is inferred that the plastic deformation decreases with the increase of the embedded depth. There is also a decrease in the change of the size of the pits, so the loss of contact is less likely to happen for larger embedded depth. The comparison of the shear stress at the surface reference point for different embedded depth is shown in Fig. 26. The amplitudes of the shear stress increase with the embedded depth and serious distortion can be observed in the shear stress curves for smaller embedded depth. A bigger amplitude of the shear stress generated by the vibrato at the ground surface is beneficial for the transmission of the shear waves to the deeper earth. Thus, we can conclude that smaller embedded depth can reduce the application of the outgoing waves and the efficiency of the exploration.

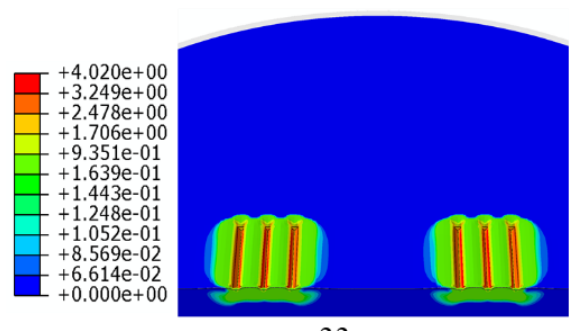

$33 \mathrm{~mm}$

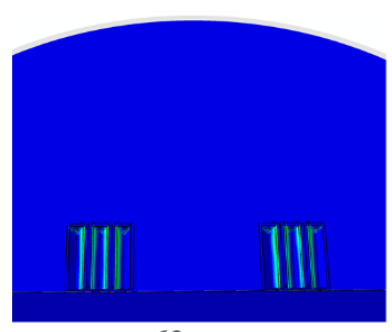

$69 \mathrm{~mm}$

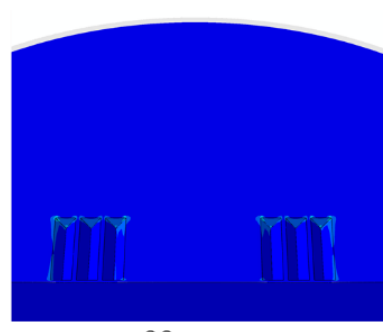

$99 \mathrm{~mm}$

Figure 25. The equivalent plastic strain for different embedded depth.

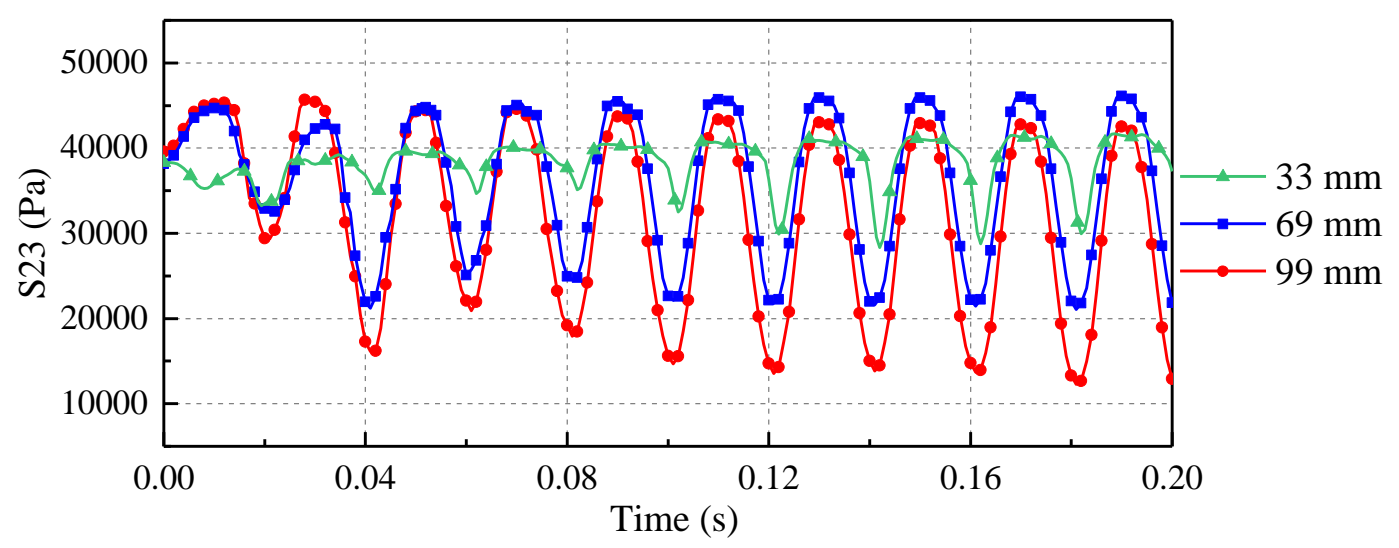

Figure 26. Comparison of shear stress for different embedded depth.

The ground force generated by the vibrator for different embedded depth is shown in Fig. 
27. It is seen that the amplitude of the ground force increases with the embedment, thus increasing the depth is a way to increase the vibrator output. Figure 28 shows the energy characteristics of the vibrator-ground system for different depths. The external work decreases with the increase of the embedded depth, so the vibrator needs more hydraulic power for lower embedment. On the contrary, the effective energy transferred to the ground increases with the embedded depth. The transferred ratio of the system also increases with the embedded depths, and the average values of the transfer ratio for $33 \mathrm{~mm}, 69 \mathrm{~mm}$, and $99 \mathrm{~mm}$ are $0.68 \%, 9.69 \%$, and $38.15 \%$, respectively. According to the above analysis, smaller embedded depth will reduce the power of the vibrator and increase the energy consumption, which has negative influence on the exploration effectiveness.

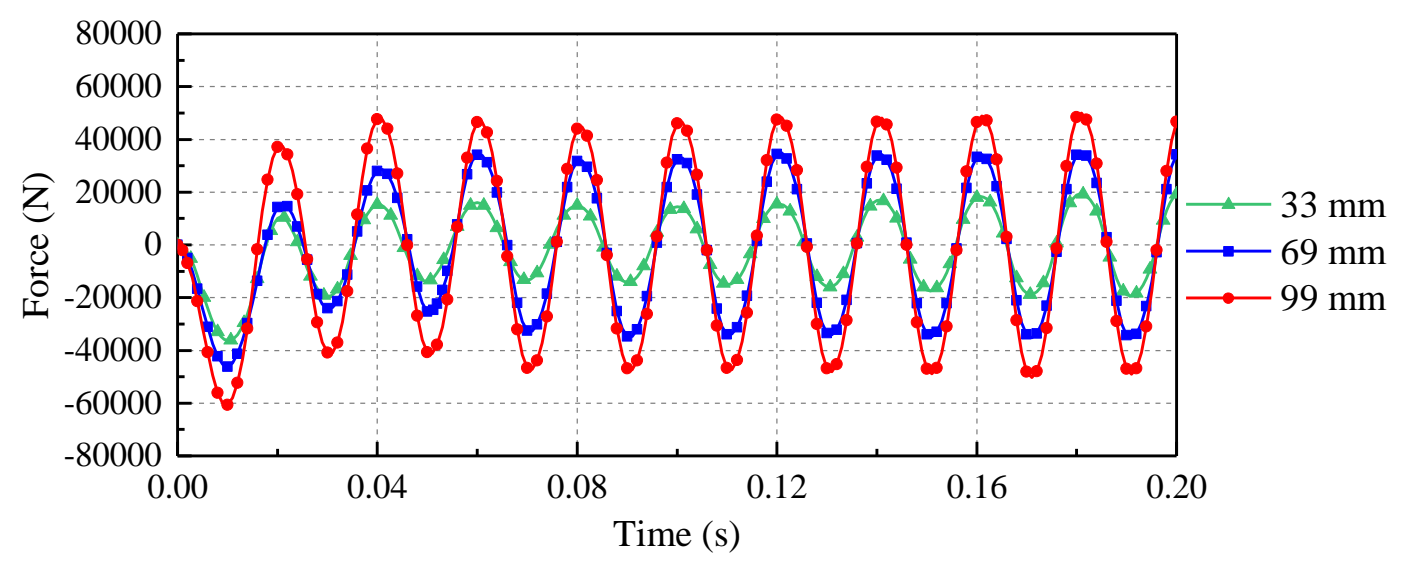

Figure 27. The ground force of the vibrator for different embedded depths.

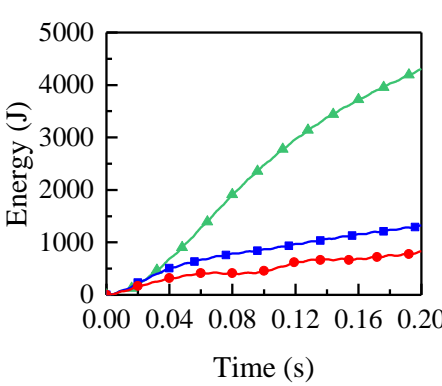

(a)

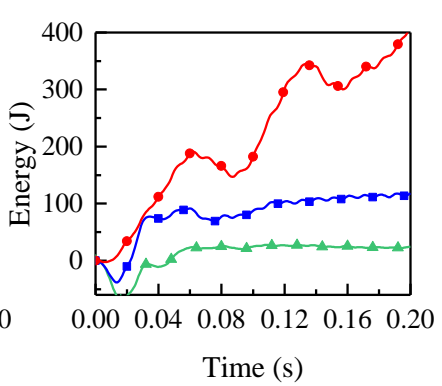

(b)

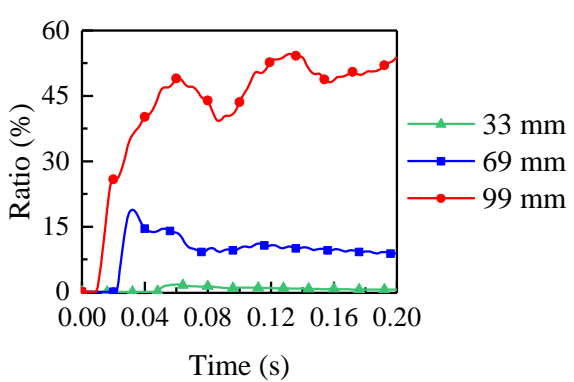

(c)

Figure 28. The energy characteristics for different embedded depths: (a) external work; (b) effective transferred energy; (c) transfer ratio.

Overall, according to the parametric analysis, it is inferred that the excitation frequency, the soil properties and embedded depths all have great influence on the performance of the vibrator. The low-frequency excitation can increase the excitation depth but generate large plastic deformation and bring about the increase of the size of the pits between the baseplates and ground. The operation in sand can decrease the ground force of the vibrator and reduce the 
signal accuracy, as well as the small embedded depth. Thus, improving the stiffness of sand by using a pre-compressional method and increase the embedded depth by optimizing the baseplates structure and installation method can be an efficient way to improve the output and efficiency of the s-wave vibrator.

\section{Conclusion}

In this paper, a 3D finite element analysis of the s-wave vibrator-ground system, considering a frictional interface between soil and baseplates and absorbing boundary conditions, is presented and discussed. A parametric analysis, to understand the influence of the frequency of the cyclic load, the type of soil and the depth of embedment, is performed, and optimal operational conditions are derived and justified, based on the stress distribution and displacements at the interfaces, the plastic deformation, the seismic wave propagation, the ground force, and the energy characteristics. The main derived conclusions are listed next:

(1) The results show that the rotation of the vibrator causes an asymmetrical stress distribution at the interfaces of the right and left baseplate. The displacement curves and the plastic deformation show that the size of the pits on the ground increases with the loading time which might bring about a contact loss at the interfaces. Both the sub harmonics and higher harmonics in the responses are observed in the generated seismic wave. According to the system energy characteristics, it is seen that the energy dissipation due to the plasticity takes the majority of the external work which makes the effective transfer ratio is low.

(2) An evaluation criterion is presented to evaluate the performance of the vibrator. The effects of the system parameters, such as excitation frequency, soil material and embedded depth on the performance of the vibrator are discussed, showing that: the vibrator can generate larger ground force and more effective transferred energy in the low-frequency phase, but the plastic deformation of the ground also increases which will bring about a lower transfer ratio. The comparison of the different soils show that the soil plasticity can reduce the vibrator power and increase the signal distortion. And the vibrator operating in sand has the smallest output as well as the accuracy of outgoing waves. Moreover, increasing embedded depth can increase the power of the vibrator and the decrease the harmonic distortion effectively.

\section{Acknowledgements}

This work was supported by the National High Technology Research and Development Program of China (Grant No: 2012AA061201) and the China Scholarship Council. 


\section{References}

[1] Wei Z, Phillips TF, Hall MA. Fundamental discussions on seismic vibrators. Geophysics 2010;75:13-25.

[2] Beresnev IA. Ground-force- or plate-displacement-based vibrator control? J Sound Vib 2012;331:1715-21.

[3] Caldwell, J., Marine multicomponent seismology, Leading Edge, 1999; 18: 1274-1282.

[4] Stewart, R. R., Gaiser, J.E., Brown, R.J. \& Lawton, D.C., Converted-wave seismic exploration: applications, Geophysics, 2002; 68:1348-1363.

[5] Martin, J. E., and Jack, I. G. The behavior of a seismic vibrator using different phase control methods and drive level: First Break, 1990;8: 404-414.

[6] Walker,D. Harmonic resonance structure and chaotic dynamics in the earth-vibrator system: Geophysical Prospecting, 1995; 43:487-507.

[7] Jeffryes BP. Far-field harmonic measurement for seismic vibrators. 66th Annual International Meeting, SEG, Expanded Abstracts, 60-63.

[8] Veen MVD, Brouwer J, Helbig K. Weighted sum method for calculating ground force: an evaluation by using a portable vibrator system. Geophys Prospect 2010;47:251-67.

[9] Wei Z. Design of a P-wave seismic vibrator with advanced performance. GeoArabia 2008;13:123-36.

[10] Saragiotis C, Scholtz P, Bagaini C. On the accuracy of the ground force estimated in vibroseis acquisition. Geophys Prospect 2010;58:69-80.

[11] Poletto F, Schlifer A, Zgauc F, et al. Borehole signals obtained using surface seismic and ground-force sensors. 81st Annual International Meeting, SEG, Expanded Abstracts, 4298-303.

[12] Castanet A, Lavergne M. Vibrator controlling system. U.S. Patent 1965:3208550.

[13] Sallas J, Weber R. Comments on "The amplitude and phase response of a seismic vibrator" by Lerwill WE, Geophys Prospect 2010;30:935-8.

[14] Sallas JJ. Seismic vibrator control and the downgoing P-wave. Seg Technical Program, Expanded Abstracts 1982;1:732-40.

[15] Lebedev AV, Beresnev IA. Nonlinear distortion of signals radiated by vibroseis sources. Geophys 2004;69:968-77.

[16] Lebedev AV, Beresnev IA, Vermeer PL. Model parameters of the nonlinear stiffness of the vibrator-ground contact determined by inversion of vibrator accelerometer data. Geophysics 2006;71:25.

[17] Noorlandt R, Drijkoningen G. On the mechanical vibrator-earth contact geometry and its dynamics. Geophysics, 2016;81:37-45.

[18] Peng X, Huang Z, Hao L. Modeling of nonlinear interaction and its effects on the dynamics of a vibrator-ground system. Soil Dyn Earthq Eng, 2020; 132:106064.

[19] Li G, Huang Z, Lian Z, Hao L. A model for the vibratror-groundc coupling vibration and 
the dyanamic responses under excitation of sweep signal, Adv Struct Eng, 2019; 22: 18551866.

[20] Baeten, G., and A. Ziolkowski, The vibroseis sources, 1990, Elsevier.

[21] Wei Z. Modeling and model analysis of seismic vibrator baseplate. Geophys Prospect, 2010; 58:19-31.

[22] Wei Z. Pushing the vibrator ground-force envelope towards low frequencies. Geophys Prospect, 2009; 57:19-32.

[23] Huang, Z., Peng, X., Li, G. Optimization of the dynamic stiffness of the seismic vibrator. Adv Mech Eng, $2018 ; 10: 1-11$

[24] Milkereit B , H. STüMPEL, Rabbel W . Shear-wave reflection profiling for near-surface lignite exploration. $1986 ; 34: 845-855$.

[25] Mueller M C . Using shear waves to predict lateral variability in vertical fracture intensity. The Leading Edge, 1992; 11:29.

[26] Lu XL, Li PZ, Chen B, Chen YQ, Computer simulation of the dynamic layered soil-pilestructure interaction system. Can Geotech J, 2005; 42: 742-51.

[27] Achmus M, Kuo Y S, Abdel-Rahman K. Behavior of monopile foundations under cyclic lateral load. Comput Geotech, 2009; 36: 725-735.

[28] Kim Y, Jeong S. Analysis of soil resistance on laterally loaded piles based on 3D soil-pile interaction. Comput Geotech, 2011; 38: 248-257.

[29] Achmus M, Akdag C T, Thieken K. Load-bearing behavior of suction bucket foundations in sand.Appl Ocean Res, 2013; 43: 157-165.

[30] Huang M, Liu Y. Axial capacity degradation of single piles in soft clay under cyclic loading. Soils and Foundations, 2015, 55: 315-328.

[31] Trochanis AM, Bielak J, Christiano P. Three-dimensional nonlinear study of piles. J Geotech Eng, 1991; 117(3): 429-47.

[32] C. Luo, X. Yang, C. Zhan, X. Jin, Z. Ding, Nonlinear 3D finite element analysis of soilpile-structure interaction system subjected to horizontal earthquake excitation, Soil Dyn Earthq Eng, 2016; 84:145-156.

[33] Naderi-Boldaji M, Alimardani R, Hemmat A, et al. 3D finite element simulation of a single-tip horizontal penetrometer-soil interaction. Part I: Development of the model and evaluation of the model parameters, Soil Till Res, 2013, 134: 153-162.

[34] Popova E, Popov V L. The research works of Coulomb and Amontons and generalized laws of friction. Friction, 2015, 3: 183-190.

[35] Lysmer J, Kuhlemeyer R. Finite dynamic model for infinite media. J Eng Mech Div ASCE, 1969; 95:859-77.

[36] Kuhlemeyer, R. L. and Lysmer, J. Finite element method accuracy for wave propagation problems. J Eng Mech Div ASCE, 1973; 99: 421-427.

[37] Cheng C Y, Dasari G R, Chow Y K, et al. Finite element analysis of tunnel-soil-pile 
interaction using displacement controlled model. Tunn Undergr Sp Tech, 2007, 22: 450466.

[38] Susana, López-Querol, Jaime, Peco, Juana, Arias-Trujillo,Numerical modeling on vibroflotation soil improvement techniques using a densification constitutive law. Soil Dyn Earthq Eng, 2014; 65:1-10.

[39] Hilber H M, Hughes T J R. Collocation, dissipation and [overshoot] for time integration schemes in structural dynamics. Earthq Eng Struct D, 1978, 6: 99-117.

[40] Karthigeyan S, Ramakrishna V, Rajagopal K. Influence of vertical load on the lateral response of piles in sand. Comput Geotech, 2006, 33: 121-131. 